\title{
Über den Nachweis von Tuberkelbazillen im aspirablen Staub.
}

\author{
Von \\ I. Engelhardt, \\ appr. Arzt. \\ Mit 10 Textabbildungen.
}

Eine wichtige Ergänzung zum Problem der Inhalationstuberkulose ist die Frage, ob denn wirklich im aspirablen Staub solche Mengen von virulenten Tuberkelbazillen (T B) vorkommen, dass damit die Häufigkeit der tuberkulösen Infektion [1-8] erklärt werden könnte.

Der Nachweis von T B im Staub wurde wiederholt erfolgreich versucht; zuerst und am gründlichsten, übrigens auch mit bisher unübertroffener Technik von $\mathrm{C}$ ornet [9] in Krankenbäusern, Wohnungen und Werkstätten. (Näheres darüber siehe weiter unten!) Nach ihm gelang der Nachweis noch sehr oft, zunächst E. Kr üger [10], dann u. a. noch den folgenden; A. Kustermann [11] in Gefängnissen, Prausnitz [12] und Petri [13] in Eisenbahnwagen, Kirchner [14] in einer Militärmontierungskammer, Deipser [15] in Schulräumen, F. Gots chlich [16] in öffentlichen Sälen, Wagner [17] in einer Lungenheilstätte, B. He y m an n [18] in Privatwohnungen und Krankensälen, Lenoir und Camus in Krankensälen, und zwar sowohl durch Injektion von Staubproben [19] als durch Halten von Kaninchen, welche in den betreffenden Räumen erkrankten [20],

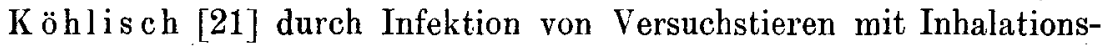
apparaten.

Aber die positiven Resultate finden sich immer nur an zweifellos stark infizierten Orten oder in nächster Nähe unreinlicher Phthisiker. Mit Cornet [22] hat man vielfach daraus geschlossen, dass auch 
nur dort infektiöser Staub sei. Betrachtet man jedoch die angewandten Methoden näher, so kann man auch zu anderen Schlüssen kommen. Cornet z. B., welcher, wie gesagt, die exaktesten Lntersuchungen zuerst und in grösster Anzahl vorgenommen bat, entnahm den aus der Luft an nicht direkt infizierbaren Stellen abgelagerten Staub mit Spatel, Pinsel oder Schwämmchen (am erfolgreichsten mit Schwämmchen, wie auch Heymanns [18] Parallelversuche bestätigen), emulgierte den Staub in Bouillon und injizierte dieselbe Meerschweinchen intraperitoneal [9]. Ein ziemlich erheblicher Prozentsatz der Tiere erlag septischen Infektionen. Erlitten haben, da der Staub völlig unbehandelt war, wohl alle eine solche, sind ihr aber nicht alle erlegen, sondern haben sie oft spurlos überstanden. Denkt man nun an die Beobachtungen von Lupusheilungen durch Erysipel, so fragt man sich, ob das von Cornet [23] für intraperitoneale Injektion von Meerschweinchen angenommene Infektionsminimum ron 43 T B nicht heraufgesetzt wird bei gleichzeitiger Einverleibung septischer Keime. Jedenfalls könnten bei jedem gesund gebliebenen Tier mindestens 42 , bei den an Wundinfektion gestorbenen vielleicht noch mehr TB dem Nachweis entgangen sein. Nehmen wir aber an, es seien in einem Raum per Schwämmchenprobe $40 \mathrm{~T} \mathrm{~B}$ (und man bekommt mit dem Schwämmchen sicher nicht alle auf der Fläche befindlichen), so besteht jedenfalls die Möglichkeit, dass das genügt, um jene Infekte zu setzen, die etwa der bekannten Naegelischen Statistik [3] zugrunde liegen. Dass derartige geringfügige Infekte bei den Versuchstieren vorgelegen haben, ist jedenfalls möglich, da es durch die dazu nötigen gründlichen und mühsamen Untersuchungen (Zerlegung aller Lymphknoten in Serienschnitte) nicht in Zweifel gestellt ist. Denn, dass auch bei Meerschweinchen ganz leichte, abheilende tuberkulöse Infektionen vorkommen, ist doch wahrscheinlich.

Würde man mit einem Material arbeiten, das von septischen Keimen befreit ist, so wären die Wundinfektionen zu vermeiden. Damit fiele der Einwand einer eventuellen Erhöhung des Infektionsminimums, die an Sepsis eingehenden Fälle fielen fort und so würden sicher mehr T B nachzuweisen sein. Das ist durch Antiforminbehandlung [24-28] des betreffenden Staubes leicht zu erzielen. Es werden dadurch bekanntlich, wenigstens bei kürzerer Einwirkung, die $\mathbf{T} B$ in ihrer Lebensfähigkeit nicht beeinträchtigt, während alle anderen Keime vernichtet und auch sonst viele organische Bestandteile aufgelöst werden.

Wie gesagt, entnahm Cornet die Staubproben von Stellen, welche einer direkten Infektion (Ausspucken und dgl.) unzugänglich 
waren, so dass der Staub sicher aus der Luft stammte und also hätte aspiriert werden können. Aber wie lange lagerte er schon? Wieviel ron den T B waren schon zugrunde gegangen? Dass dieselben sehr empfindlich sind und im trockenen Staube oft rasch absterben, zeigen die Untersuchungen Kirsteins [30]. Cornet sagt, die Quantität des untersuchten Staubes habe den Niederschlag aus über 50000 Litern Luft repräsentiert und sei daher mehr gewesen, als ein Mensch in über 100000 Atemzügen hätte einatmen können. So überzeugend das klingt, so wahrsuheinlich ist es doch auch, lass das Sediment der gewaltigen Luftmenge durch längeres Lagern bedeutend an Virulenz eingebüsst hat. Also ein Bild von der T B-Virulenz des momentan aspirablen Staubes hat Cornet nicht gegeben. Denn was uns jeweils gefährden kann, ist doch nicht nur der Staub hinter dem Kopfende des Bettes und an den übermannshohen Partien der Wände, sondern es ist aller Staub, der jeweils durch Luftbewegung aufgewirbelt und aspiriert werden kann.

Es wäre daher wünschenswert, grössere Proben, z. B. das zusammengefegte Material eines Raumes zu untersuchen. Wäscht man dies in Antiforminlösung, so ist ausserdem anzunehmen, dass die an groben Partikeln haftenden Bakterien losgelöst und in die Waschflüssigkeit aufgenommen werden, so dass man auf eine grosse Menge groben Staubes verzichten und sich mit dem Sediment des Antiforminwaschwassers begnügen kann.

Es bleibt aber immer noch der Nachteil, dass man lauter Material untersucht, das vielleicht niemals za Inhalationsinfektion hätte führen können, weil es weder durch Luftströmung aufgewirbelt, noch aspiriert worden wäre. Auch wenn man mit einem Schwämmchen oder dgl. eine Fläche abreibt, so erhält man ein ganz anderes Material, als was je auf natürlichem Wege in den Respirationtraktus gelangen könnte. Nicht ob irgendwo in einem Staub irgendwelcher Herkunft T B sind, ist ja das zu lösende Problem, sondern, ob im a spirablen Staub welche nachgewiesen werden können. Es gilt also in einem Raume allen Staub aufufangen, der der Aspiration zugänglich ist, bzw. ihn zu aspirieren, z. B. mit einem Staubsaugapparat, den man so einrichtet, dass seine Saugkraft etwa ebensoviel ausrichtet, wie die natürliche Aufwirbelung (Wind, Durchzug, Fegen usw.) und die Einatmung.

Findet man dann im so aspirierten Staub nach Antiforminbehandlung wirklich oft und in grösserer Menge als bisher nachgewiesen 'T B, so darf man annehmen, dass die so überaus verbreiteten, meist geringfügigen tuberkulösen Infektionen zum grossen Teil durch bazillenhaltigen Staub entstehen können. 
Man würde so einerseits der Lösung der allgemeinen ätiologischen Frage nach dem Infektionsmodus, anderseits der Lösung des mikrobiologischen Problems von der Verbreitung und eventuellen Ubiquität der T B näher kommen. Endlich würde, wenn auf diesem Wege etwa eine entscheidende Antwort auf die Ubiquitätsfrage gegeben werden könnte, die sozialhygienische Streitfrage geklärt werden, ob die Isolierung der Kranken oder die Massnabmen zur Therapie und Prophylaxe der konstitutionellen tuberkulösen Disposition schliesslich wirksamer sein müssen im Kampfe gegen die Tuberkulose.

Es kam nun zunächst darauf an, eine Methode zu erproben, um in einer Portion Staub etwaige T B nachzuweisen.

Dass der biologische Nachweis durch intraperitoneale Injektion bei Meerschweinchen am feinsten ist, darf mit Cornet [29] angenommen werden. Natürlich durfte die Menge des Injektionsmaterials und seine Grobkörnigkeit nicht so gross sein, dass allein der mechanische Reiz das Tier gefährdete. Aus diesem Grunde ist die Staubmenge, die ohne weiteres durch direkte Injektion untersucht werden kann, begrenzt, während es in unserem Falle sehr vorteilhaft erschien, durch Auswaschen mit Antiforminlösung und Verwendung des Waschwassersedimentes viel bedeutendere Staubmengen der Untersucbung zugänglich zu machen.

Es wurde zunächst eine T B-Emulsion hergestellt durch Verreibung einer im Dampftopf sterilisierten Serumagarkultur mit physiologischer Kochsalzlösung unter Zusatz von $1 \%$ Karbolsäure. Die Färbung von Ösenausstrichen dieser Emulsion ("E") ergab zwar, dass dieselbe sehr ungleichartig war, neben vielen einzelnen Stäbchen noch grössere zusammenhängende Rasen enthielt. Hatte die Emulsion aber einige Zeit gestanden, so senkten sich die Rasen zu Boden und in dem Oberteil fanden sich noch stundenlang in gleichmässiger Verteilung einzelne Stäbchen. Nach drei Stunden konnten noch so viele nachgewieden werden, dass eine sebr langsame Spontansedimentierung der TB angenommen werden musste, und daher vermutet wurde, auch in dem Antiforminwaschwasser würden sich dieselben noch lange schwebend erhalten, nachdem der Staub sich bereits gesenkt.

Hierauf wurden ca. $40 \mathrm{ccm}$ zusammengefegten Fussbodenstaubes aus dem Laboratorium des Bakteriologischen Untersuchungsamtes mit $5 \mathrm{ccm} \mathrm{E}$ feucht rermischt und getrocknet. Dann wurden $3 \mathrm{ccm}$ dieses Staubes („S 1“) mit $22 \mathrm{ccm} 20^{\circ} \%$ Antiforminlösung kräftig geschüttelt; bald setzte sich am Boden des Glases der nunmehr sauber aussehende Staub ab und zwar nach der Korngrösse geschichtet, der feinste Staub oben, während einige spezifisch leichte 
organische Teile, wie Haare, Holzsplitter und dgl. an der Oberfläche schwammen. Zwischen Bodensatz und Oberfläche fand sich eine trüb homogene, gelbbräunliche Flüssigkeit, in der nun die TB zu erwarten waren.

Es wurde daher nach etwa einhalbstündigem Stehen von der homogenen Zwischenschicht $20 \mathrm{ccm}$ herauspipettiert, zentrifugiert, das Zentrifugat gewaschen, mit verdünnter Eiweisslösung ausgestrichen und nach $Z i e h l$ gefürbt. Trotz vielfacher sorgfältiger Wiederholung und Verkürzung der Sedimentierungszeit auf wenige Minuten, fanden sich niemals T B im Präparat.

Der mit T B versetzte Staub wurde nun mit aller Vorsicht nocheinmal („S 2") hergestellt, aber die Resultate wiederholter färberischer Nachweisversuche blieben negativ.

Also konnten sich die T B tatsächlich nicht in der Zwischenschicht befinden. Nach Analogie der Bakterienfällung mit Eisenchlorid und Ammoniumkarbonat war zu vermuten, dass dieselben relativ schnell, vielleicht vermöge besonderer Adsorptionsfähigkeit durch die feinsten Staubteile niedergerissen wurden. Die gröbsten Partikel sanken schon in ca. 1/2 Sekunde zu Boden. Die bei dieser Fallgeschwindigkeit bestehende Reibung der Partikeloberfläche an der Flüssigkeit musste anhaftende Bakterien wohl sicher abstreifen, die Reibung musste die Adsorptionskraft überwiegen $(R>A)$, während bei kleinem, ziemlich langsam (also mindestens erst nach einigen Sekunden) sinkendem Staube wohl die Reibung geringer sein konnte als die Adsorptionskraft $(\mathrm{R}<\mathrm{A})$, so dass die Bakterien mitgenommen wurden. Wann aber, bei welcher Korngrösse bzw. bei welcher Fallgeschwindigkeit lag die Grenze zwischen beiden Extremen, wann war $\mathrm{R}=\mathrm{A}$ ?

Bei den Versuchen mit S 1 und S 2 war bereits die Sedimentierungszeit soweit verkürzt worden, bis im mikroskopischen Präparat wegen der grossen schwarzen Staubteile die Erkennung ron 'T B unmöglich war. Wie gesagt, hatten sich in keinem Falle T B nachweisen lassen.

Um nun einmal die Hypothese nachzuprüfen, die T B würden durch feinen Staub sehr bald, durch groben nicht sedimentiert, wurde folgender Versuch gemacht:

Durch Aussieben und Ausschwemmen von Streusand wurde eine Portion ganz reinen Sandes gewonnen, der sich schon in ein bis zwei Sekunden vollständig sedimentierte, wobei das Oberteil ganz klar blieb („S 3"). Es erübrigt sich, zu bemerken, dass die. Klärung des Sandes durch Auswaschen mit Salzsäure bedeutend erleichtert wurde. 
I. $3 \mathrm{ccm} \mathrm{S} 3$ wurden mit $0,5 \mathrm{ccm} \mathrm{E}$ und $21,5 \mathrm{ccm}$ Leitungswasser kräftig geschüttelt, das Oberteil 3 Minuten nach Absetzung des Sedimentes zentrifugiert und das Zentrifugat ausgestrichen und gefärbt.

II. $2 \mathrm{ccm}$ Calcium carbonicum praecipitatum wuràen mit $0,5 \mathrm{ccm} \mathrm{E}$ und $22,5 \mathrm{ccm}$ Leitungswasser kräftig geschüttelt, das Oberteil nach 3 Minuten zentrifugiert und das Zentrifugat, welches allerdings noch viel Kalk enthielt, ausgestrichen und gefärbt.

III. $0,5 \mathrm{ccm}$ E wurden mit $24,5 \mathrm{ccm}$ Leitungswasser kräftig geschüttelt und nach 3 Minuten langem Stehen wurden die oberen $20 \mathrm{ccm}$ zentrifugiert und das Zentrifugat ausgestrichen und gefärbt.

Resultat: I und III zeigen etwa das gleiehe Bild: zahlreiche säurefeste Stäbchen. In II sind keine Bakterien nachweisbar, aber wegen der vielen Kalkstäubchen ist das Bild sehr unklar.

Darauf wurde der ganze Versuch wiederholt, wobei jedoch die Sedimentierungszeit auf $1 / 4$ Stunde rerlängert wurde, so dass das Oberteil von II ziemlich geklärt war. $\mathrm{Zu}$ dem letzteren wurden dann einige Tropfen verdünnter Salzsäure zugesetzt, sodass es vollkommen klar wurde. Die gleiche Menge Salzsäure erhielten um der Gleichmässigkeit willen die Oberteile von I und III. Das Resultat der mikroskopischen Untersuchung war prinzipiell das gleiche: In I und III viele, wenn auch etwas weniger säurefeste Stäbchen, in II nichts.

Hierauf wurden die Kalksedimente von II beider Versuche in verdünnter Salzsäure vollständig aufgelöst, die spezifisch sehr schwere Lösung mit Methylalkohol ungefähr wieder auf das spezifische Gewicht des Leitungswassers gebracht, zentrifugiert und das Zentrifugat wiederholt gewaschen, dann ausgestrichen und gefärbt: zahlreiche säurefeste Stäbchen im mikroskopischen Bild beider Sedimente.

Mithin stand es fest, dass die T B tatsächlich durch feinen Staub in sehr kurzer Zeit sedimentiert werden konnten.

Jetzt handelte es sich nur noch darum, festzustellen, wie lange man eine Staubmischung verschiedenster Korngrösse stehen lassen durfte, ohne dass nemnenswerte Mengen der enthalteinen T B zu Boden sanken, also welcher Zeitpunkt der Grenze $\mathrm{R}=\mathrm{A}$ entsprach. Dann würde es jedenfalls genügen, kurz vor diesem Zeitpunkte abzugiessen und das Oberteil ca. 10 Minuten nochmals stehen zu lassen, worauf das nunmehrige zweite Sediment die T B enthalten musste. So musste man dann auch das Auswaschen mit einfacher Spontansedimentierung erzielen können; das Zentrifugieren des Staubwassers würde in Zukunft nicht mehr nötig sein.

In Ergänzung zu den letzten Versuchen wurde noch der folgende gemacht:

$2 \mathrm{ccm} \mathrm{S} 3$ wurden mit $1 \mathrm{ccm}$ Kalkstaub, $0,5 \mathrm{ccm} \mathrm{E}$ und $21,5 \mathrm{ccm}$ Leitungswasser kräftig geschüttelt, das Oberteil nach 3 Minuten langem Stehen abgegossen, 
mit Salzsäure geklärt, zentrifugiert usw.; im mikroskopischen Präparat keine TB. Das Sediment wurde nun mit verdünnter Salzsäure völlig vom Kalkniederschlag befreit, mit Methylalkohol versetzt, nach 3 Minuten Jangem Stehen dus nunmebrige Oberteil zentrifugiert, gewaschen usw.; im mikroskopischen Präparat viele TB.

Es stand also ausser allem Zweifel, dass es der feine Staub war, welcher die T' B zu Boden zog, dass diese dagegen ohne solchen Staub sich längere Zeit schwebend erhielten.

Inzwischen wurde der geeignete Staubsaugapparat fertiggestellt. Als ein saugkräftiger, leicht transportabler Handbetriebs-

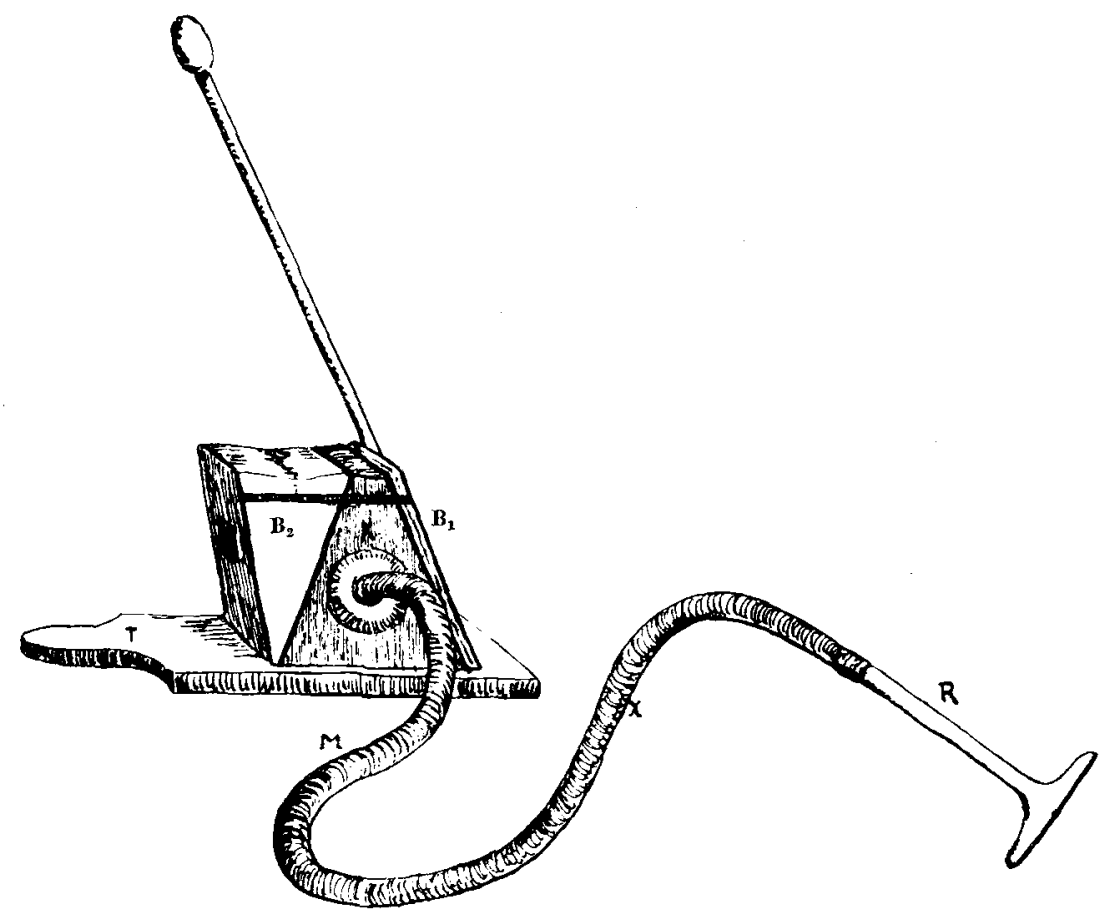

Fig. 1.

apparat gelangte der Daisy-Apparat von Abner zur Verwendung, der durch den Fabrikanten liebenswürdigst zur Verfügung gestellt wurde (Fig. 1).

Er bestebt aus einer Kammer (K) von der Form eines dreiseitigen, auf einer Haupttäche liegenden Prismas, welche auf einer breiten hölzernen, mit einem Trittbrett ( $\mathrm{T}$ ) als Widerhalt versehenen Unterlage befestigt ist. An jeder der beiden anderen Hanptflächen der Kammer ist ein Blasebalg von gleicher Form und Grösse angebracht. Die beiden Blasebälge (B1, B2) sind äusserlich durch Schienen so miteinander verbunden, dass sie nur gleichzeitig bewegt werden können und der eine komprimiert ist, wenn der andere sich dehnt. An B1 befindet sich ein langer Hebel, durch welchen das Gebläse in Betrieb gesetzt wird. Jeder 
Blasebalg ist mit der Kammer durch eine Ventilöffnung verbunden, welche nur Luft in der Richtung von $K$ nach $B$ durchlässt, mit der Aussenwelt durch

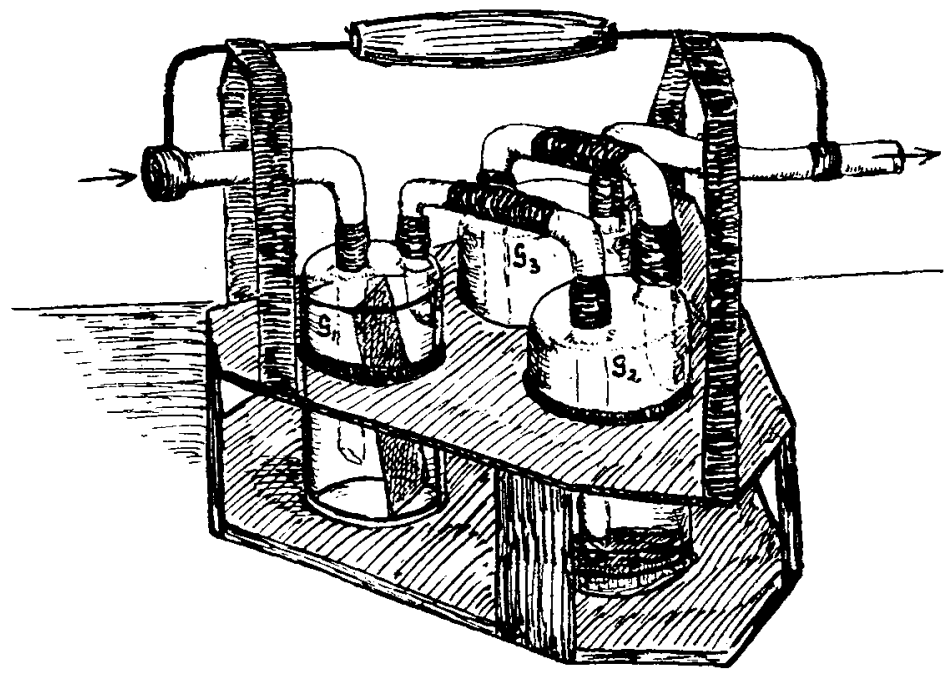

Fig. 2.

eire Ventilöffnnng, welche nur Luft binaus, aber nicht hereinlässt. So sangt also B 1 bzw. B 2 bei Ausdehnung Luft aus $K$ an und treibt sie bei Kompression ins Freie. In der Kammer befindet sich ein Sack, in welchen der Metallschlauch (M)
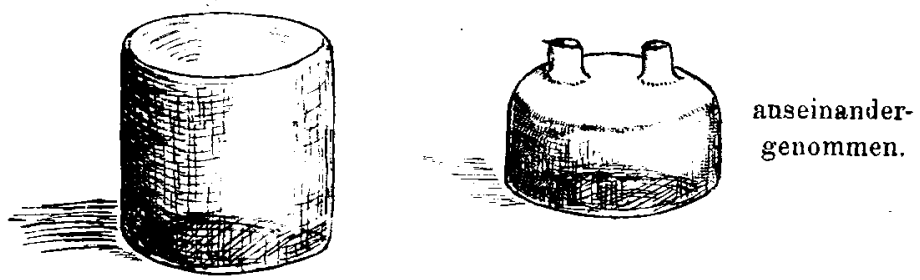

$G_{1}$ oder $G_{2}$ ohne $\mathrm{Zu} \cdot$ bzw. Ableitungsröhren.

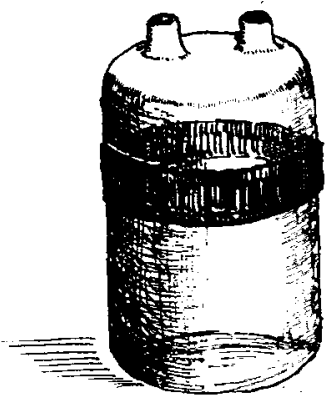

mit Guınmigürtel zusammengesetzt.

Fig. 3. 
von der staubaufsaugenden Röhre (R) führt. Der Sack sollte im gewöhnlichen Betrieb zum Auffangen des Staubes dienen. Als tür unsere $\mathrm{Zwecke}$ ungenügend konnte er entfernt werden. Um dagegen den Staub quantitativ aufzufangen, wurde in den Metallschlauch bei $\mathrm{X}$ ein System eingeschaltet, das nach vielfachem Ausprobieren verschiedener Möglichkeiten folgende Konstruktion aufweist: In einem Holzrahmen mit Griff (Fig. 2) sind drei gleichartige Glasflaschen (G1, G 2, G 3) befestigt. Die Flaschen haben je zwei Öffnungen, durch welche die Zu- und Ableitung mit eingesetzten, durch überzogene Gummischläuche befestigten Glasröhren vermittelt werden. $G 1$ und $G 2$ sind in $2 / 3$ Höhe horizontal gespalten (Fig. 3) und durch einen Gummigürtel zusammengehalten. GI (Fig. 4) ist durch ein eingesetztes, an einem Drahtrahmen befestigtes, schräggestelltes Drahtsieb in zwei Abteilungen geteilt (Fig. 5), welche je dem Zuleitungs- bzw. Ableitungsrohr entsprechen. Aus dem Metallschlauch gelangt die staubhaltige Luft zunächst durch

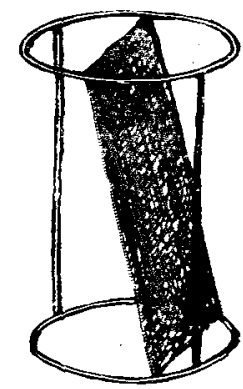

Drahtnetzeinsatz von $G_{1}$.

Fig. 4.

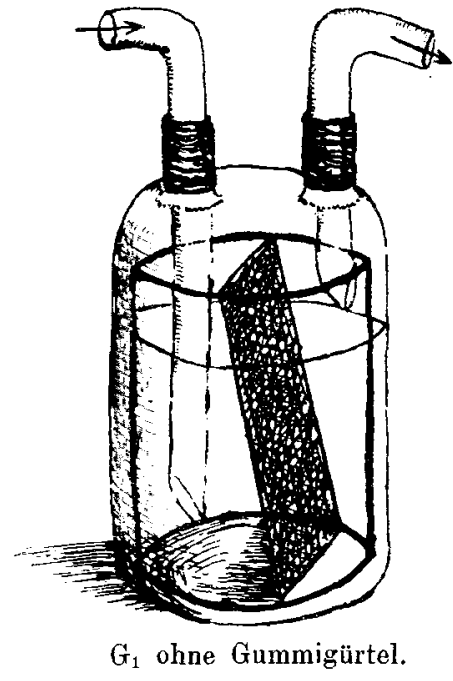

Fig. 5.

das längere Zuleitungsrohr in den unteren Teil von G1. Hier verteilt sich'der bisher kräftige Luftstrom auf einen grösseren Raum, verlangsamt sich daher und lässt viele gröbere Partikel fallen, ausserdem hält das Drahtsieb Haare, Fäden und dgl., welche später bei der Injektion die Hoblnadel verstopfen, zurück. Die Stromverlangsamung in $G 1$ geht gerade soweit, dass sie dem natürlich vorkommenden Luftstrom entspricht; das Drahtsieb hält leichte, aber doch umfangreiche Teile zurück, welche z. B. bei der Atmung die Nase sicher nicht passieren würden. Durch das kurze Ableitungsrohr wird die Luft aus $G 1$ fort und nach G 2 gefïhrt, wo sich ein Einsatz befindet (Fig 6 und 7), welcher zwei parallel und horizontal angeordnete, ca. $3 \mathrm{~cm}$ voneinander entfernte Drahtnetze enthält, von denen das untere ca. $3 \mathrm{~cm}$ über dem Boden bleibt; ferner befindet sich Wasser darin, dessen Spiegel bis zu halber Höhe zwischen beiden Drahtnetzen reicht. Die aus $G 1$ kommende Luft gelangt nun durch oin langes Zuleitungsrohr, welches beide Drahtnetze darchbohrt, auf den Boden von G2 und gibt an das Wasser fast allen Staub ab, während die grossen Luftblasen durch die Drahtnetze 
zersplittert werden und daher ohne grosse Blasenbildung die Luft oben durch die wieder kurze Ableitungsröhre nach G3 überführt werden kann. Das Zuleitungsrohr von $\mathrm{G} 3$ reicht bis an das untere Drittel der Flasche, welche im übrigen nichts besonderes enthält; das Ableitungsrohr ist wieder kurz und geht direkt in den zum Saugapparat führenden Metallschlauch über. $G 3$ hat lediglich den Zweck, ganz kleine Wassermengen, deren Hinüberspritzen sich nicht ver-

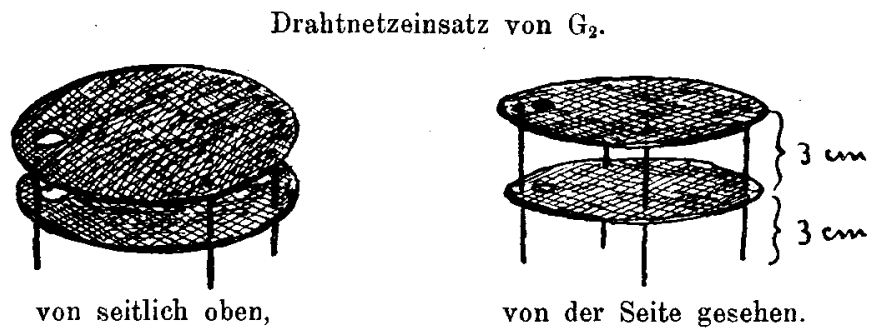

Fig. 6.

meiden lässt, aufzunehmen, damit der Saugapparat trocken bleibt. Wie gesagt, hat G1 nar den Zweck, den nicht aspirationsfähigen Staub zurückzuhalten. Es befand sich also der aspirable Staub im Wasser von G2.

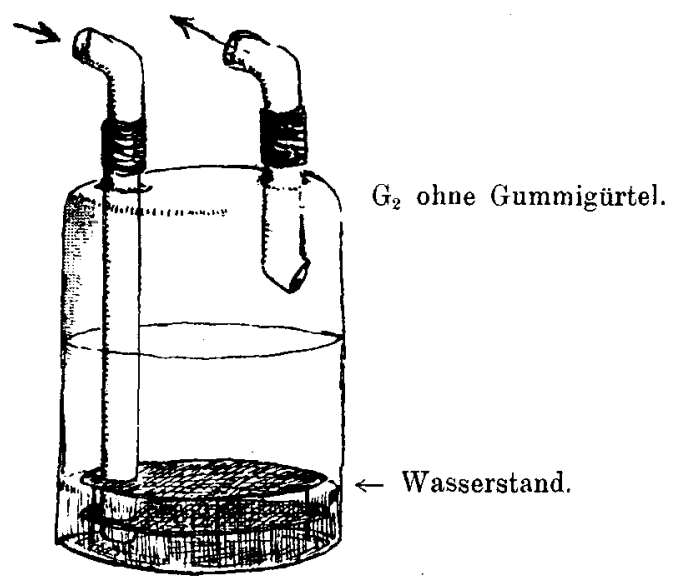

Fig. 7.

Auf diese Weise wurden in staubigen Kellerräumen des Pathologischen Institutes grössere Portionen Staub gewonnen, mit denen festgestellt werden sollte, wie lange ein Staubwasser der Spontansedimentierung überlassen werden durfte, ohne dass die enthaltenen TB in wesentlicher Menge zu Boden sanken, ferner, ob der in dieser Zeit sedimentierende Staub überhaupt so umfangreich war, dass sich eine Ausschaltung lohnte.

Da es sich hier um ein quantitatives Problem handelte, nämlich um die Frage, wieviel TB nach bestimmter Zeit noch im Oberteil 
waren, kam nur mikroskopischer Nachweis mit eventueller Auszählung der T B in Betracht. Um nun den Übelstand zu vermeiden (siehe Seite כ), dass die Erkennung von TB im mikroskopischen Präparat wegen vorhandener Staubteile unmöglich war, wurde der Staub durch fraktioniertes Ausschwemmen zunächst in vier Partien getrennt, welche je nach 10, 20,60 bzw. 120 Sekunden dauernder Spontansedimentierung das Oberteil fast vollkommen klar liessen. Dieses sogenannte fraktionierte Ausschwemmen wurde folgendermassen gemacht. Eine Portion Staubflüssigkeit wurde in einem Zylinderglas (Fig. 8), welches am Boden nach einer Seite eine stiefelförmige Aus-

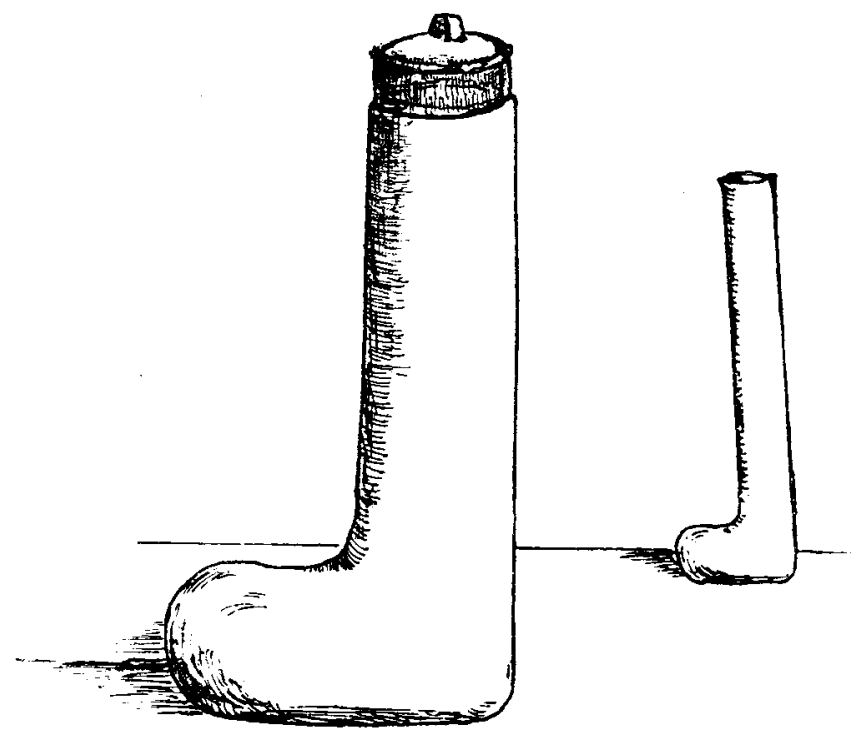

Fig. 8 .

Fig. 9.

stülpung trägt, geschüttelt, mit der verschlossenen öffnung nach abwärts gehalten, dann plötzlich umgedreht, richtig auf den Tisch gestellt und je nachdem 10, 20,60 oder 120 Sekunden stehen gelassen. Dann wurde das Oberteil abgegossen, wobei das Sediment in der stiefelförmigen Ausstülpung verblieb. Mehrfach wurde dann wieder reines Wasser aufgegossen und die gleiche Manipulation mit der gleichen Sedimentierungszeit wiederholt bis schliesslich das Oberteil nahezu klar blieb ${ }^{1}$ ). So war eine Staubart gewonnen, welche sich genau in der betreffenden Zeit sedimentierte, aber von langsamer sedimentierenden Verunreinigungen frei war, so dass das Oberteil nahezu klar blieb. In dem letzteren befindliche T B konnten also aus-

1) Das endgültige Sediment wurde dann getrocknet und sterilisiert. 
zentrifugiert, ausgestrichen, gefärbt und ungestört mikroskopisch beobachtet werden.

Wenn man also eine wässerige T B-Emulsion mit solchem Staube versetzte, musste sich leicht feststellen lassen, ob und wieviel $\mathrm{T} \mathrm{B}$ derselbe bei seiner Sedimentierung mitriss. Diese betreffenden Staubarten sollen im folgenden mit St 10, St 20, St 60 und St 120 bezeichnet werden.

Leichter als mit Hilfe von Kulturen wurde eine grössere Menge wässeriger $1 \%$ Karbol mit $0,8 \mathrm{NaCl}$-haltiger T B-Emulsion („E 1 “) hergestellt aus Sputum von der Phthisiker-Abteilung der medizinischen Klinik, welches mit Antiformin homogenisiert und dann zentrifugiert wurde.

Damit ein ungefährer quantitativer Vergleich der gewonnenen T B-Mengen möglich war, wurde das Zentrifugat in den folgenden Versuchen jedesmal durch Eiweiss-Wasserzusatz auf ein Volumen von $0,2 \mathrm{ccm}$ gebracht und dann in der Höhlung eines hohlgeschliffenen Objektträgers (Hohlschliffdurchmesser $16 \mathrm{~mm}$ ) gleichmässig verteilt und angetrocknet. Der Hohlschliff erschwerte die mikroskopische Besichtigung nur wenig; an dem ausgiebigeren Gebranch der Mikrometerschraube gewöhnte man sich leicht. Bei einfachem Ausstrich von $0,2 \mathrm{ccm} \mathrm{E} 1$ fanden sich so durchschnittlich $700 \mathrm{~T} \mathrm{~B}$ in $1 \mathrm{qmm}$.

Jetzt wurden 6 am Ende mit der genannten stiefelförmigen Ausstülpung verseheue, zum Stehen eingerichtete Reagenzgläser (Fig. 9) in folgender Weise gefüllt (Vergl. die Übersicht der Abkürzungen Seite 18):

Nr. 1. 0,2 ccm E 1 ad 25,0 ccm phys. Na Cl-Lösung.

Nr. 2. $3 \mathrm{ccm} \mathrm{S} 3$ und $0,2 \mathrm{ccm} \mathrm{E} 1$ ad $25,0 \mathrm{ccm}$ phys. Na Cl-Lösung.

Nr. 3. $3 \mathrm{ccm}$ St 10 und $0,2 \mathrm{ccm} \mathrm{E} 1$ ad $25,0 \mathrm{ccm}$ phys. $\mathrm{NaCl}-$ Lösung.

Nr. 4. $3 \mathrm{ccm}$ St 20 und $0,2 \mathrm{ccm} \mathrm{E} 1$ ad $25,0 \mathrm{ccm}$ phys. $\mathrm{NaCl}$ Lösung.

Nr. 5. $3 \mathrm{ccm}$ St 60 und $0,2 \mathrm{ccm} \mathrm{E} 1$ ad $25,0 \mathrm{ccm}$ phys. NaClLösung.

Nr. 6. $3 \mathrm{ccm}$ St 120 und $0,2 \mathrm{ccm} \mathrm{E} 1$ ad $25,0 \mathrm{~cm}$ phys. $\mathrm{NaCl}$ Lösung.

Sie wurden dann geschüttelt und 4 Minuten stehen gelassen, das Oberteil jedes Glases abgegossen, auf 3 Zentrifugiergläschen verteilt, zentrifugiert, das Sediment jedes Gläschens auf $0,2 \mathrm{ccm}$ gebracht (mit Eiweisswasser), auf einem Hohlschliffobjektträger angetrocknet und gefärbt, so dass von jeder Nr. (1-6) 3 Präparate vorhanden waren. An jedem Präparate wurden mindestens 3 Zählungen 
eines qmm ausgeführt, so dass für jede Nr. 9 Zählungen vorlagen, deren Durchschnitt folgendes Bild ergab, nachdem die betreffenden Zahlen, die ja einem Präparate, daher einem Drittel jeder Nr. entsprechen, verdreifacht sind :

Nr. 1. (Zentrifugat des Abgusses nach 4 minutenlangem Stehen von $0,2 \mathrm{E} 1$ mit 25,0 phys. $\mathrm{Na}$ Cl-Lösung) $225 \mathrm{~T} \mathrm{~B}$ in $q \mathrm{~mm}$.

Nr. 2. (Zentrifugat des Abgusses nach 4 minutenlangem Stehen von 0,2 E1 mit 25,0 phys. NaCl-Lösung und 3,0 S3) 204 T B in qmm.

Nr. 3. (Zentrifugat des Abgusses nach 4 minutenlangem Stehen von 0,2 E 1 mit 25,0 phys. NaCl-Lösung und 3,0 St 10) 90 TB in $\mathrm{qmm}$.

Nr. 4. (Zentrifugat des Abgusses nach 4 minutenlangem Stehen von 0,2 E 1 mit 25,0 phys. NaCl-Lösung und 3,0 St 20) 33 TB in $\mathrm{qmm}$.

Nr. 5. (Zentrifugat des Abgusses nach 4 minutenlangem Stehen von 0,2 E1 mit 25,0 physikalischer Na Cl-Lösung und 3,0 St 60) 0,6 T B in $\mathrm{qmm}$.

Nr. 6. (Zentrifugat des Abgusses nach 4 minutenlangem Stehen von 0,2 E1 mit 25,0 phys. NaCl-Lösung und 3,0 St 120) 0 T B in qmm.

Bei direktem einfachem Ausstrich der 0,2 E 1 fanden sich, wie wir sahen, $700 \mathrm{~T} \mathrm{~B}$ im qmm wieder.

Bei Nr. 1 mussten also die fehlenden 475 teils beim Umgiessen verloren gegangen, teils in den 4 Minuten sedimentiert sein.

Nr. 2 zeigt wieder, dass der reine grobe Sand keine nennenswerten Mengen T B mitreisst.

Dagegen beweisen die Nr. 3-6, dass schon in kürzester Zeit die meisten $\mathrm{T} B$ rom Staube mitgerissen werden, und dass 10 Sekunden Sedimentierzeit zu lang sind.

In der oben angegebenen Weise wurde nun eine neue Serie von Staubarten gewonnen und zwar mit einer Sedimentierungsdauer von 3. 5 und 8 Sekunden: St 3, St5, St 8 , wobei es ziemlich zeitraubend war, die genügende Menge St 3 zu gewinnen. Dann wurden wieder 6 stiefelförmige Reagenzgläser gefüllt, geschüttelt usw., jedoch wurden sie nur 2 Minuten stehen gelassen.

Das Resultat war das folgende:

Nr. 1 (Zentrifugat des Abgusses nach 2 minutenlangem Stehen von 0,2 E 1 mit 25,0 phys. NaCl-Lösung) $519 \mathrm{~T} \mathrm{~B}$ in $1 \mathrm{qmm}$.

Nr. 2. (Zentrifugat des Abgusses nach 2 minutenlangem Stehen von $0,2 \mathrm{E} 1$ mit 25,0 phys. NaCl-Lösung und 3,0 S3) $543 \mathrm{~TB}$ in $1 \mathrm{qmm}$. 
Nr. 3. (Zentrifugat des Abgusses nach 2 minutenlangem Stehen von 0,2 E 1 mit 25,0phys. Na Cl-Lösung und 3,0 St3) 549 'T B in 1 qmm.

Nr. 4. (Zentrifugat des Abgusses nach 2 minutenlangem Stehen von $0,2 \mathrm{E} 1$ mit 25,0 phys. Na Cl-Lösung und 3,0 St 5) $507 \mathrm{~T} \mathrm{~B} \mathrm{in} 1 \mathrm{qmm}$.

Nr. 5. (Zentrifugat des Abgusses nach 2 minutenlangem Stehen von $0,2 \mathrm{E} 1$ mit 25,0 phys. NaCl-Lösung und 3,0 St 8) $339 \mathrm{~TB}$ in $1 \mathrm{qmm}$.

Nr. 6. (Zentrifugat des Abgusses nach 2 minutenlangem Stehen von $0,2 \mathrm{E} 1 \mathrm{mit} 25,0$ phys. NaCl-Lösung und 3,0 St 10) $228 \mathrm{~TB}$ in $1 \mathrm{qmm}$.

Wegen der, wenn auch geringen und vielleicht wegen unvermeidlicher technischer Ungenauigkeiten unkorrigierbaren Unstimmigkeiten der Nr. 1-3, deren Resultate eher in umgekehrter Folge zu erwarten waren, wurde nun diese Versuchsserie wiederholt mit folgenclem Resultat:

Nr. 1. (Zentrifugat des Abgusses nach 2 minutenlangem Stehen von $0,2 \mathrm{E} 1$ mit 25,0 phys. $\mathrm{NaCl}$-Lösung) $597 \mathrm{~T} \mathrm{~B}$ in $1 \mathrm{qmm}$.

Nr. 2. (Zentrifugat des Abgusses nach 2 minutenlangem Stehen von $0,2 \mathrm{E} 1$ mit 25,0 phys. NaCl-Lösung und 3,0 S3) 561 T B in 1 qmm.

Nr. 3. (Zentrifugat des Abgusses nach 2 minutenlangem Stehen von $0,2 \mathrm{E} 1$ mit 25,0 phys. Na Cl-Lösung und 3,0 St 3) $573 \mathrm{~T} \mathrm{~B}$ in $1 \mathrm{qmm}$.

Nr. 4. (Zentrifugat des Abgusses nach 2 minutenlangem Stehen von $0,2 \mathrm{E} 1$ mit 25,0 phys. Na Cl-Lösung und 3,0 St 5) 555 T B in 1 qmm.

Nr. 5. (Zentrifugat des Abgusses nach 2 minutenlangem Stehen von 0,2 E 1 mit 25,0 phys: NaCl-Lösung und 3,0 St 8) $327 \mathrm{~T} \mathrm{~B} \mathrm{in} 1 \mathrm{qmm}$.

Nr. 6. (Zentrifugat des Abyusses nach 2 minutenlangem Stehen von $0,1 \mathrm{E} 2$ mit 25,0 phys. $\mathrm{Na}$ Cl-Lösung und 3,0 St 10) $243 \mathrm{~T} \mathrm{~B}$ in $1 \mathrm{qmm}$.

Wenn auch die beiden letzten Versuchsserien keineswegs restlose Übereinstimmung aufweisen, so zeigen sie doch deutlich zunächst das eine, dass die $\mathrm{Nr}$. 1-3 nicht wesentlich voneinander abweichen und dass mit St 3 eher noch weniger $\mathrm{TB}$ sedimentiert werden als mit S3 bzw. in ganz reinem Wasser.

Zwar sind in St 3 die gröbsten Staubteile überhaupt enthalten, aber quantitativ ist es nur sehr wenig, was damit ausgeschaltet wird, so dass es $z$ weifelhaft erscheint, ob sich diese Mühe lohnt.

St 5 dagegen stellt schon einen grösseren Anteil des ursprünglichen Staubes dar. Allerdings wird auch dadurch die Sedimentierung der TB, wenn auch nur wenig, nämlich um ca. $5,5 \%$, vermehrt. $5,5 \%$ der $\mathrm{T} B$ würden also etwa dem Nachweis entgehen, wenn der sich in 5 Sekunden sedimentierende Staub ausgeschaltet wird. Um jedoch zu entscheiden, ob etwa die Gefährdung der Tiere durch St 5 und die eventuell damit verbundene Nachweiserschwerung mehr ins Gewicht fällt als die genannten $5,5 \%$, hätte es weiterer umständlicher Versuche bedurft, auf die zunächst verzichtet wurde. Viel- 
mehr wurde kurzweg beschlossen, den 5 -Sekundenstaub auszuschalten. Denn bedeutsam konnte doch der TB-Verlust von $5,5 \%$ nur werden, wenn die Zahl der T B ohnehin hart an der Grenze der Nachweisbarkeit st:ınd. Dagegen wurde die Injektionstechnik, wie entsprechende Versuche bald zeigten, sehr durch den Fortfall relativ gröberer Staubelemente erleichtert. Cornet [9] z. B. musste, um den Staub injizieren zu können, eine so dicke Injektionsnadel anwenden, dass die Injektionswunde durch einen umstochenen Faden geschlossen werden musste und öfter auch Eiterung der Wunde eintrat. Bei den weiter unten geschilderten Versuchen konnten dagegen nach Ausschaltung von St 5 genügend feine Injektionsnadeln verwendet werden, um ron einer besonderen Schliessung der Wunde abzusehen. Bei Injektion des nach Cornet gewonnenen Materials genügte zwar auch oft die feine Nadel, aber mehrfach mussten gröbere Kaliber verwendet werden, bei denen allerdings auch immer von besonderen Massnahmen zur Schliessung der Wunde abgesehen wurde und nur einmal (Tier Nr. 1) äusserliche Eiterung auftrat. Diese jedenfalls im Anfang der Tierversuche deshalb, weil infolge geringer Übung der Einstich nicht glatt und schnell genug erfolgte.

Als praktischstes und indifferentestes Medium zum Behandeln des Untersuchungsmaterials wurde im folgenden nicht reines Wasser, sondern phys. NaCl-Lösung verwandt.

Mithin war nun die anzuwendende Methode des TB-Nachweises im aspirablen Staub die folgende:

1. Reinigen des zu untersuchenden Raumes mit dem angegebenen Staubsaugapparat, dessen Saugrohr und Staubauffangsytem sterilisiert sind.

2. Das Staubwasser der mittleren Flasche ( 62 Seite 9) wird mit $20 \%$ Antiformin versetzt, 15 Min. im Stiefelglas (Seite 11) geschüttelt, 10 Min. stehen gelassen, Oberteil vom Sediment abgegossen; dann das Sediment mit steriler phys. NaCl-Lösung 6 mal durchAufgiessen, Schütteln, 10 Min. stehen lassen und Abgiessen gewaschen. Darauf wird das Sediment in ein steriles Reagenz-Stiefelglas getan,mit phys. $\mathrm{NaCl}-L o ̈ s u n g ~ g e s c h u ̈ t t e l t$ und nach 5 Sek. abgegossen; dies erste $\tilde{5}$ Sek.-Sediment jedoch noch 3 mal mit phys. NaCl-Lösung wieder aufgeschüttelt und abgegossen; alle 4 Abgüsse dann zusammengetan und $1 / 2$ Stunde stehen gelassen; das nun nach nochmaligem Abguss bleibende Sediment auf $15 \mathrm{ccm}$ mit phys. NaCl-Lösung aufgefüllt, um zur Injektion verwendet $\mathrm{zu}$ werden.

3. Die $15 \mathrm{ccm}$ Injektionsmaterial werden zu gleichen Teilen drei möglichst gleichartigen gesunden Meerschweinchen intraperitoneal injiziert. 
Es kam nun darauf an, die angegebene Methode, welche im folgenden kurzweg die Methode nach E. genannt werden soll, auf einige Fälle anzuwenden und dabei mit der bisher erfolgreichsten, der Methode nach Cornet, zu vergleichen.

Die letztere Methode [9] wurde so ausgeführt, dass mit einem sterilen feuchten Augenschwamm, welcher in einer sterilen Aluminiumdose aufbewahrt und mit einer sterilen Sekond-Zange gefasst wurde, die hinter dem Kopfende eines bettlägerigen Patienten befindliche Wand, die am Kopfende des Bettes vorhandenen Querleisten, wenn sie an die Wand anstiessen, ferner hochhängende Bilder und Uhrgehäuse und dgl. solange abgewischt wurden, bis der Schwamm keinen Staub mehr aufzunehmen vermochte; dass dann ein solcher Schwamm, in der sterilen Metalldose heimtransportiert, in $15 \mathrm{ccm}$ steriler Kulturbouillon unter aseptischen Kautelen, d. h. von einer mit sterilem Gummihandschuh versehenen Hand, ausgewaschen und alsdann die Bouillon zu gleichen Teilen drei möglichst gleichartigen, gesunden Meerschweinchen intraperitoneal injiziert wurde.

Um die Überlegenheit einer der beiden Methoden einwandfrei sicher zu stellén, bedarf es natürlich vieler hundert Anwendungen. Selbstverständlich soll daher den folgenden wenigen Versuchen gar keine ausschlaggebende Bedeutung beigelegt werden. Dieselben wurden vielmehr nur angestellt, um in der Kürze der Zeit wenigstens einen ungefähren Anhalt zu gewinnen, ob eine weitere Nachprüfung der Methode nach E. aussichtsvoll ist oder nicht.

Einiges Allgemeine ist noch iiber die folgende Wiedergabe der Versuchsprotokolle zu sagen. Nach Bedarf und Interesse finden sich Angaben über: „Familienverhältnisse“, d. h. Art, Zahl und Gesundheit der Wohnungsgenossen; "Wohnverhältnisse", d. h. Art, Zahl, Verwendung und Mietpreis der Wohnräume; "Krankengeschichte“, d. h. kurzer Bericht über Entwickelung und Stand des tuberkulösen Leidens des polikinischen Patienten. Zur Untersuchung wurden herangezogen: die Wohnräume von 3 offenen Phthisikern, welche alle einigermassen reinlich waren, $\mathrm{Na}, \mathrm{Gu}, \mathrm{Ka}$; ferner der Wohnraum einer reinlich behandelten Gelenktuberkulose, St; der Wohnraum eines unreinlichen Phthisikers, We; der sehr schmutzige und unhygienische von ganz verkommenen, aber anscheinend gesunden Hinterbliebenen bewohnte Raum eines vor 4 Wochen verstorbenen offenen, sehr unsauberen Phtisikers, $\mathrm{Ke}$; allerdings war dieser Raum gleich nach dem Tode amtlich nit Formalin desinfiziert worden ${ }^{1}$ ); endlich der sehr saubere Wohnraum

1) Vgl. dazu Bauer Bothel, „Über widersprechende Erfahrungen mit Formalindesinfektion bei Tuberkulose". Zeitschr. f. Tbc. XVI. 3. 1910. 
eines völlig Gesunden, En; und der Kurssaal des pathologischen Instituts, Ku. Unter "Raum" sind regelmässig kurze Angaben zur hygienischen Einschätzung des untersuchten Raumes gemacht. Nach

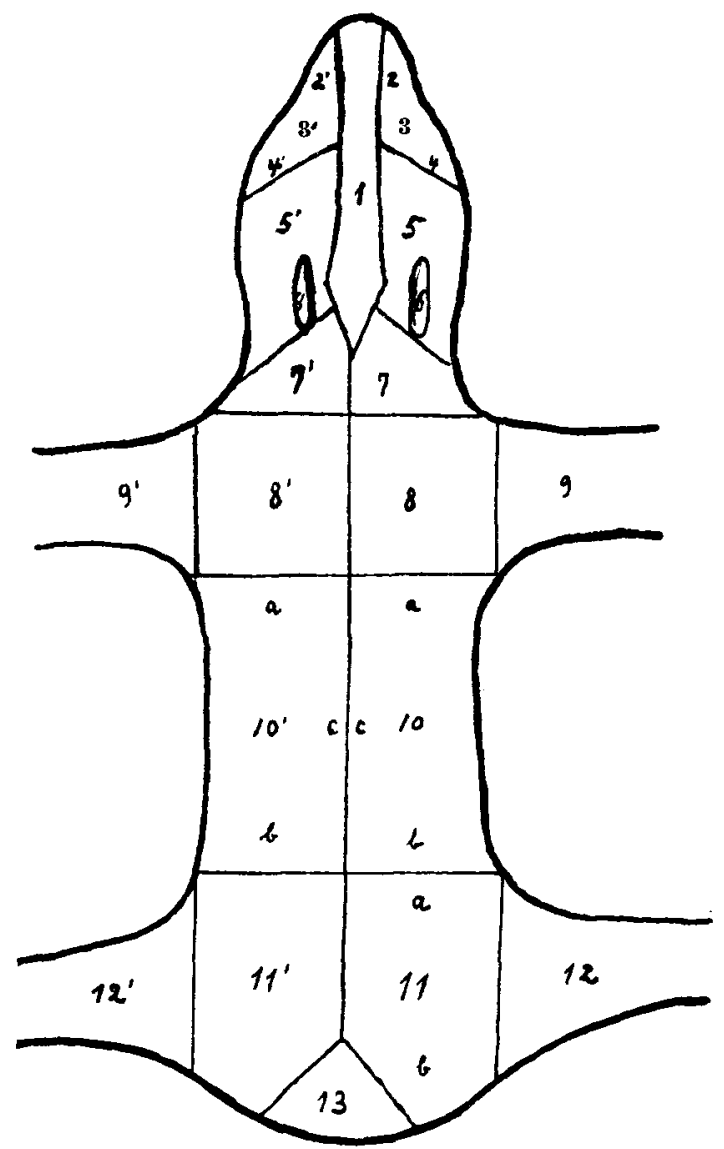

Schematische Felderung der Dorsalseite eines Meerschweinchens.

Bauchfläche 14

vord. Brustfläche 15

3 u. $3^{\prime}=$ Augen

6 u. $6^{\prime}=$ Ohren $\mathrm{K}=1-6$ u. $2^{\prime}-6^{\prime}$

$$
\begin{aligned}
& \mathrm{W}=2-4 \text { oder } \mathrm{W}^{\prime}=2^{\prime}-4^{\prime} \\
& \mathrm{Th}=7-9 \text { and } 7^{\prime}-9^{\prime} \\
& \mathrm{Ru}=10 \text { und } 10^{\prime} \\
& \mathrm{Hi}=11-13 \text { und } 11^{\prime} \text { u. } 12^{\prime}
\end{aligned}
$$

Fig. 10.

Angaben über die Entnahme des Staubes nach Corn et und nach E. folgt dann das Injektionsdatum und sind die Identitätsmerkmale der Versuchstiere nach nebenstehendem Schema (Fig. 10) angegeben. Endlich folgen die Sektionsbefunde. Zur Diagnose wurden makroskopi- 
scher, histologischer und bakteriologischer Befund verwertet. Als diagnostische Resultate wurden in Betracht gezogen:

Tbc. mit Bazillenbefund T

Tbc. ohne Bazillenbefund.

Tod an Sepsis

überstandene Sepsis

nichts besonderes nachweisbar $\mathrm{N}$

missglückter Versuch

\section{Übersicht über die Abkürzungen.}

$\mathrm{T} \mathrm{B}=$ Tuberkelbazillen.

Die in [ ] gesetzten Zahlen beziehen sich auf das Literaturverzeichnis am Schluss.

$\mathbf{E}=\mathrm{T}$ B-Kultur-Emulsion in physiolog. Kochsalzlösung mit $1 \%$ Karbolsäure (Seite 4).

S 1 = Zusammengefegter Fussbodenstaub mit einem Zusatz von E vermischt und getrocknet (Seite 4).

S 2 = Ebenfalls zusammengefegter Fussbodenstaub mit einem Zusatz von $E$ vermischt und getrocknet (Seite 5).

S 3 = Geklärter, rasch sedimentierender Sand (Seite 5).

St $10=$ Derart ausgeschwemmter Kellerstaub, dass er in Wasser nach 10 Sekunden ohne Trübung sedimentiert (Seite 12).

St $20=$ Dsgl. nach 20 Sekunden sedimentierend (Seite 12).

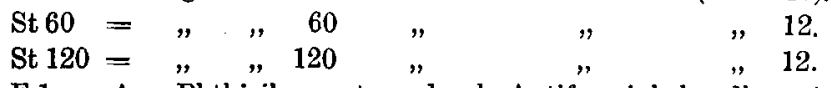

E $1=$ Aus Phthisikersputum durch Antiforminbehandlung herge tellte T BEmulsion in physiolog. Kochsalzlösung mit $1 \%$ Karbolsăure (Seite 12).

St $3=$ Derart ausgeschwemmter Kellerstaub, dass er in Wasser nach 3 Sekunden ohne Trübung sedimentiert (Seite 13).

St $5=$ Dsgl. nach 5 Sekunden sedimentierend (Seite 13).

St $8=", \quad " \quad$ " " 13 .

Die zur besseren Übersichtlichkeit angewandten Abkürzungen der Versuchstier-Sektionsdiagnosen sind sowohl auf dieser Seite wie auf Seite 27 erklärt.

\section{Versuchsprotokolle.}

1. Vorversuch am 1. Dezember 1911.

$5 \mathrm{ccm}$ tbc. Sputum mit $10 \mathrm{ccm} 25 \%$ Antiformin ca. $1 / 2$ Std. gesehüttelt, zentrifugiert, mit $0,9 \%$ Kochsalzlösung gewaschen, zentrifugiert, in $5 \mathrm{ccm} 0,9 \%$ steriler Kochsalzlösung.

Schwarz, w., 7', 9', 12' weiss, 6, 13 gelb.

Injiziert intraperitoneal. (bloss subkutan geraten!)

Sektion am 16. Januar 1912:

An der Injektionsstelle : kirschgrosser, abgekapselter, subkutaner Abszess mit gelblich-weissem Eiter.

Inguinaldrüsen hart vergrössert, Axillardrüsen dsgl.

Peritoneum und Netz anscheinend frei. Einige hart vergrösserte Mesenterialdrüsen. Milz und Leber von miliaren bellen Herden durchsetzt, besonders 
die Milz, die stark vergrössert und höckrig ist. Nieren, Nebennieren, Darm u, a. anscheinend frei.

Herz o. B. Isungen enthalten beide im oberen und mittleren Teil ausgedehnte graue Infiltrate. Mediastinaldrüsen hart vergrössert.

Ausstrich des Abszesseiters: Lympho- und Leukozyten, keine Bakterien im Präparat. Der gesamte Eiter (1-2 ccm) mit $20 \%$ Antiformin behandelt und zentrifugiert; im Ausstrich des Zentrifugates zahlreiche säarefeste Stäbchen.

In Formol-Müller eingelegt: Mehrere Drüsen, Milz, l. Niere, Teile der 1. Lunge, Abszesswand.

2. Na. 13. Dezember 1911.

Familienverhältnisse: ca. 40 Jahre alte Tochter und deren ca. 2 Jahre altes Kind, beide angeblich gesund.

Wohnverhältnisse: alle schlafen im gleichem $R$ a um in verschiedenen Betten. Ausserdem noch Küche verhanden mit Loggia nach Süden. Mietpreis 19 Mark.

Krankengeschichte: 77 Jahre alte Frau mit doppelseitiger progredienter Phthise und T B im Sputum.

Raum: Vierter Stock nach Norden. Schlaf- und Wohnzimmer, 3 Betten, von Pat. nachts und ca. ${ }^{1 / 2}$ Tag benutzt. Ziemlich' sauber. 250 hoch, 460 tief, 500 breit; ein Fenster 120: 160; direkte Horinzontbeleuchtung.

Nach Cornet: Obere Leiste von drei Bildern über dem Bett, Rückwand des Bettkopfendes und ca. 1 qm Wand oben über dem Bett.

Nach E. ca. 3 qm der Bettwand, Fussbodenwandwinkel, Fussbodenritzen, Teppich, 4 Bilder.

Nach Cornet, 13. Dez.:

Nr. 1, ganz grau, m.

Nr. 2, ganz schwarz, w.

Nr. 3, gelb, w., 1 schwarz, $2^{\prime} 4^{\prime}$ und $10^{\prime}-10$ grau-schwarz.

Nach E., 14. Dez.:

Nr. 4, grau, w., 1 und 14 weiss.

Nr. 5, weiss, w., $1-4$ und $2^{\prime}, 4^{\prime}, 11^{\prime}$ schwarz, 11 gelb.

Nr. 6, gelb, w. 1,12 und $12^{\prime}$ weiss, 8,9 und $8^{\prime}$ schwarz.

23. XII. Nr. 1 hat eiternde Einstichstelle.

Nr. 3 gestorben; Sektion: grosser abgekapselter, subhepatischer AbszesB (im Ausstrich Diplokokken), vergrösserte Milz. - sept. Infektioin. S.

15. I. 1912: Nr. 1 getötet. Sektion: Staub in der Bauchhöhle, Verwachsung des Darms mit der vord. Bauchwand, mesenteriale Lymphdrüsen wenig hart vergrössert (Durchschnitt o. B.), Milz fleckig narbig verändert, - überstandene sept. Infektion. ( $S$.)

Nr. 2 getötet. Sektion: Staub subkutan; Inguinaldrüsen o. B.; auch sonst o. B. - nichts nachweisbar. $\mathrm{N}$.

Nr, 4 getötet. Sektion: Peritoneum o. B., einzelne unregelmässige helle Herde in Milz und Leber, einige stark vergrösserte, zentral erweichte Mesenterialdrüsen (Ausstrich: feine Gramnegative, nicht säurefeste Stäbchen und sarzineartige grobe Streptokokken); Lungen o. B.; auch sonst o. B. - überstandene sept. Infektion. (S.)

(Staub i. Bauchh.!)

17. I. Nr. 5 getötet. Sektion: subkutaner abgekapselter Abszess mit Staubresten; im Ausstrich schöne säurefeste Stäbchen; Inguinaldrüsen hart vergrössert, r. verkäst; einige hart vergrösserte Mesenterialdrüsen; vergrösserte Milz mit 
punktförmigen hellen Flecken; vergrösserte Bronchialdrüsen; infiltrierte Partien der I. Lunge; sonst 0. B. - tuberkulöse Infektion durch den Staub. T.

Nr. 6 getötet. Sektion: Staub subkutan; sonst o. B. - nichts nachweisbar. N. Resultat: C.: $(S)$ N S. $\quad$ E.: $(S)$ T N.

\section{Ke. 16. Dezember 1911.}

Familienverhältnisse: Ehemann ca. 40 Jahre alter heruntergekommener Säufer, Stuhlflechter, früher Unteroffizier, zwei Kinder, 3 und 5 Jahre alt, angeblich gesund, unterernährt aussehend.

Wohnverhältnisse: Wohnraum mit 2 Betten, in denen alle schlafen, das eine Bett im Alkoven, dazu eine Küche. Mietpreis $15 \mathrm{Mk}$. Feucht und kalt.

Krankengeschichte: 30 jährige Frau, Mai bis November 1911 in Behandlung. Seit 17. Juni in der jetzigen Wohnung.

Phthisis progressiva, l. Oberlappen und r. Spitze, zahlreiche T B im Sputum, unsauber, unfolgsam. Gestor ben Ende Nov. 1911. Städtische Desinfektion des Raumes.

Raum: Parterre, nach Osten gegen Schlossberg. Schlaf-Wohnzimmer mit 2 Betten, von Pat. Tag und Nacht benutzt. Ausserordentlich schmutzig, Stroh, Zigarrenstummel etc. am Boden. 290 hoch, 340 breit, 400 tief; zwei Fenster ì $160: 90$, davon 1 meist mit geschlossenem Laden.

Nach Cornet: Oberleiste von 2 Bildern über dem Bett, Rückwand des Bettkopfendes und ca. 1 qm Wand über dem Bett; Schwamm völlig mit Staub beladen.

Nach E.: Fussboden.

Nach Cornet, 16. Dez.:

Nr. 7, gelb, w., 1 u. $12^{\prime}$ weiss, 2, 4, 2' $4^{\prime}$ schwarzgelb, 11' 12 schwarz.

Nr. 8, struppigweiss, w. $3,3^{\prime}$ rot, 5, 5' grauschwarz, 12 schwarz.

Nr. 9, weiss, w., 9 schwarz.

Nach E.: 17. Dez. 1911.

Nr. 10 , weiss, w., $5,5^{\prime}$ schwarz.

Nr. 11, weiss, m., k, 10' schwarz, 11 gelb.

Nr. 12, weiss m., 3, $3^{\prime}$ rot, $8,11^{\prime}$ gelb, 11 grau.

17. I. 1912: Nr. 7 getötet: ebenso 8-12; die Sektionen ergeben:

Nr. 7: Haut in Umgebung der Kinstichstelle sehnig mit der Bauchwand verwachsen; darin kleiner Abszessrest (im Ausstrich nur Leukozyten) und Staubteile; Inguinaldrüsen kaum vergrössert; Brust- und Bauchhöhle o. B.; nur Milz etwas vergıössert und höckerig, mit einer narbigen Einziehung. - Überstandene sept. Infektion. $(S)$

Nr. 8: Staub in der Bauchhöhle; Magen und Leber sauber verwachsen; infiltrierte Partien in der linken unteren Lunge; vergrösserte, aber nicht erweichte Bronchialdrüsen; sonst alles o. B. - Nichts sicheres nachweisbar. N.

Nr. 9: Staub suhkutan; infiltrierte linke lungenspitze; sonst alles o. B. nichts nachweisbar. $\mathrm{N}$.

Nr. 10: Staub nur subkutan; infiltrierte Partien in der linken oberen Lunge; vergrösserte Bronchialdrüsen; sonst alles o. B. - Nichts nachweisbar. N.

Nr. 11: Staub in der Bauchböhle; im Peritoneum der Bauchwand und des Magens verstrente Knötchen mit rötlichen Verfärbungen; Netz knollig verdickt mit käsig erweichten Zentralpartien (im Ausstrich säurefeste Stäbchen); einige hart vergrösserte Mesenterialdrüsen; Milz und Leber vergrössert, punktförmig gefleckt, rauh stark vergrösserte mediastinale Drüsenpakete ohne Erweichung; in beiden 
Lungen grössere infiltrierte missfarbige Partien. - Tuberkulöse Infektion, wahrscheinlich durch den Staub. T.

Nr. 12: Subkutaner Abszess mit Staubresten; im Ausstrich nicht säurefeste Stäbchen und Diplokokken; Inguinaldrüsen vergrössert, nicht verkäst. - Überstandene sept. Infektion. (S.)

Resultat: C.: $(S)$ N N $\quad$ E.: N T $(S)$.

4. Kurssaal des Pathologischen Institutes. 3. Januar 1912.

Raum: II. Stock nach Norden (botanischer Garten), hell und luftig. 2- bis 3 mal wöchentlich zur Demonstration frischer Präparate, zweimal wöchentlich zum mikroskopischen Kurs benutzt.

Ziemlich sauber.

Nach Cornet: Oberleiste der Tafel, Oberfläche des Wandbrettes, ca. 3 qm Wandfläche in der Nahe der Tafel.

Nach E.: Fussboden und Fensterbank der Tafelgegend.

Nach Cornet: 3. Januar:

Nr. 13: weiss, w., 4, 5' $10^{\prime}$ gelb.

Nr. 14: schwarz, w. 5, $9^{\prime}, 12,14$ weiss.

Nr. 15: weiss, m. 2, 4-7 graugelb, $2^{\prime} 4^{\prime}-6^{\prime}, 13$ gelb.

Nach E.: 3. Januar:

Nr. 16: weiss, w. $3,4^{\prime}, 11$ schwarz, 13,11 a gelb.

Nr. 17: schwarz, w. 1 weiss, 2 ' 6, 9, 10 gelb.

Nr. 18: schwarz, m., 1, 2', 5'-7', 10' weiss, 4', 10 gelb.

5. I.: Nr. 15 gestorben, Sektion: peritonitisches Exsudat mit Diplokokken und feinen Stibchen; Staub in der Bauchhöhle; sonst o. B. - Sept. Infektion. S.

Nr. 14 gestorben, Sektion: gleicher Befund wie bei 15. - S.

31. I.: Nr. 13 getötet, Sektion: Staubteile in der Bauchhöhle; strangförmige Verwachsung des Darms mit der vorderen Banchwand; Verwachsung von Magen und Leber mit Abszess (im Ausstrich Diplokokken); Mjlz höckerig vergrössert; einfach verdickte Mesenterialdrüsen; abgekapselter Abszess in der rechten mittleren Lunge (im Ausstrich Diplokokken); sonst o. B. -- Überstandene sept. Infektion. (S.)

Nr. 16--18 getötet, Sektionen ergeben:

Nr. 16: Staub in der Bauchhöhle, sonst alles o. B. $-\mathrm{N}$.

Nr. 18: Befund wie bei 16 . $-\mathrm{N}$.

Nr. 17: Einstichwunde eiternd; Bauchwandabszess (Ausstrich schöne säurefeste Stäbchen und feine kurze Bazillen); stark vergrösserte, etwas verkäste Inguinaldrüsen; in der Bauchhöhle Staub; Knoten im Netz und Mesenterium; Milz und Leber vergrössert und knötchenhaltig; sonst alles o. B. - Tuberkulöse Infektion durch den Staub. T.

$$
\text { Resultat: C.: (S) S S E.: N T N. }
$$

5. Gu. 12. Januar 1912.

Familienverhältnisse: Ehefrau und 2 drei und vier Jahre alte kränkliche Kinder.

Wohnverhältnisse: ein Wohnzimmer, wo Patient schlät und sich tags meist aufhält; ein Schlafzimmer mit 2 Betten für Ehefrau und Kinder; eine kleine Küche. Preis Mk. 29.-

Krankengescbichte: 33 Jahre alt, Maler, seit 2 Jahren krank. Phthisis progressiva beider Oberlappen mit Pleuritis, reichlich Bazillen im Sputum. 
Raum: 2. Stock nach Norden, enge Gasse, ziemlich dunkel. Wohnzimmer von Patient tagstaber benutzt.

Nicht sehr schmntzig, angeblich zweimal wöchentlich geputzt und alle 14 Tage mit Chlorkalk aufgewaschen, zuletzt vor 10 Tagen.

Nach Cornet: Oberlejste von 6 Bildern und Gardinenleisten.

Nach E.: Fussboden und Lambrileiste.

Nach Cornet: 13. Januar:

Nr. 19 , weiss, w. $1-6,3^{\prime} 5^{\prime} 9^{\prime} 10^{\prime}$ schwarz.

Nr. 20, weiss, w. 4 gelb, 13 gelbbraun.

Nr. 21, weise, m. 4, 4' dankelgelb, 10 gelb und schwarz.

Nach E.: 13. Januar:

Nr. 22, weiss, m. 2, 4, 5, 4', 11, 12 schwarz, 11' schwärzlich braun.

Nr. 23, weiss, m. 2, 4-7, gelb und schwarz, 10 b, 11 a gelb, 13 gelbbraun.

Nr. 24, srhwarz, m. 1, 10', $11^{\prime} 12^{\prime}$ weiss, $10 \mathrm{~b}, 11$ a gelb.

15. I.: Nr. 19-20 gestorben: Sektionen:

Nr. 19: Staub in der Bauchböhle; trübes peritoneales Exsadat (im Ausstrich zahlreiche plumpe und feinere Stäbchen, wenig Leukozyten); Leber trúbe, matt; sonst o. B. - Sept. Infektion. S.

Nr. 20: Gleicher Befund wie 19, jedoch im Ausstrich mehr Leukozyten und nur ganz kurze, kokkenartige Stäbchen. - S.

19. I.: Nr. 23 gestorben; Sektion: Staub in der Bauchhöhle; peritoneales, leicht gerötetes und getrübtes Exsudat (Ausstrich sehr leukozytenreich, wenig kurze Stäbchen); subhepatischer Abszess und Leberinfarkt, in deren Abstrich die gleichen Stäbchen; Milz etwas vergrössert; sonst o. B. - Sept. Infektion. S.

19. II.: Nr. 21, 22, 24 getötet; Sektionen ergeben:

Nr. 21: Staub in der Bauchhöhle; vergrösserte, deformierte, fleckige, höckerige Milz; feine, versprengte Knötchen in der Leber, abgekapselter Abszess in linken Leberlappen (im Abstrich einige feine kurze, vicht säurefeste Stäbchen; Netz knotig verdickt; hart vergrösserte Mesenterialdrüsen, eine verkäst (im Ausstrich wenige säurefeste Stäbchen); sonst o. B. - Überstandene sept. und tuberkulöse Infektion durch den Staub. $(S) \mathrm{T}$.

Nr. 22: Staub in der Bauchhöhle; Knötchen im Bauchwandperitoneum; kleine Knoten im Netz; etwas vergrösserte harte Mesenterialdrüsen; nichts verkäst; sonst o. B. - Wahrscheinlich tuberkulöse Infektion durch den Staub. (T?). Stäck der Bauchwand und Drüsen in M üller-Formol eingelegt.

Nr. 24: Staub in der Bauchhöhle; Knötchen im Peritoneum der Bauchwand; miliare helle Knötchen in der Milz; im verdickten Netz grosser, verkäster Knoten mit Magen verwachsen (im Ausstrich vereinzelte, säurefeste Stäbchen sowie eigenartige, nicht säurefeste Granula); vergrösser te Bronchialdrüsen und kleinere infiltrierte Lungenpartien; sonst o, B. - Tbc. Infektion d. d. Stb. T.

Resultat: C.: S, S, $(S)$ T. $\quad$ E.: $\mathrm{S},(\mathrm{T}), \mathrm{T}$

6. We. 20. J anuar 1912 .

Familienverhältnisse : ca. 36 Jahre alte Ehefrau und 2 Kinder, 7 and 5 Jahre alt, alle angeblich gesund.

Wohnverhältnisse: Schla fzimmer mit zwei Betten, in denen alle schlafen, ausserdem Wohnzimmer und Küche. Mietpreis Mk. 20.-.

Krankengeschichte: 29 Jahre alt. Seit Herbst 1910 krank; seit Sommer 1911 in Behandlung. Doppelseitige progrediente Phthise mit TB im Sputum. Ferner Darmtuberkulose. Fast ständig bettlägerig. 
Raum: 4. Stock, nach SW, ziemlich hell, Schlafzimmer, in dem Pat. meist bettlägerig.

Ziemlich schmutzig, angeblich zweimal wöchentlich geputzt, seit einigen Tagen nicht mehr.

275 hoch, 275 breit, 560 tief. 1 Fenster $65: 85$.

Nach Cornet: Oberleiste von 5 Bildern und Balkenoberfläche, sehr staubig.

Nach E.: Fussboden und Bettrorleger.

Nach Cornet: 20. Januar:

Nr. 25, weiss, m. 4, 4', 11 gelb.

Nr. 26, gelb, w., 1, 7, 15 weiss, $11,10 \mathrm{c}$ und $10^{\prime} \mathrm{e}$ schwarz.

NB. etwas verschüttet, daher nur 2 Tiere injiziert.

Nach E.: 22. Januar 1912.

Nr. 27, weiss, w., 4. 8'-10', braun, $2^{\prime} 4^{\prime}-7^{\prime}$ schwarzbraun.

Nr. 28, braun, w., 1, 10' weiss, 4-7, 11 schwarz.

Nr. 29, struppig weiss, w., 4, 4' $10,10^{\prime}$ gelb, 11, 11' schwarz (gravid?)

24. I. : Nr. 26 gestorben; Sektion: gangränöse Einstichstelle; Staub in der Bauchhöhle; peritonitisches Exsudat rötlich trübe (im Ausstrich viele Leukozyten, viele nicht säurefeste Stäbchen); Leber brüchig, ebenso wie Milz mit feinem abstreichbarem Belag; sonst o. B. - Sept. Infektion. S.

29. I.: Nr. 25 gestorben; Sektion; grosser Abszess an der Einstichstelle, mit Darm verwachsen, sich zwischen Leber und Magen fortsetzend (im Ausstrich viele Leukozyten und nicht säurefeste Stäbchen). Sept. Infektion. S.

19. II.: Nr. 27-29 getötet; Sektionen :

Nr. 27: Feine verstreute Knötchen an dem Peritoneum der Bauchwand; Staub in der Bauchhöhle; Knoten im Netz und Mesenterium; punktförmig gefleckte, vergrösserte Milz; nirgends käsige Erweichung; ein Stück Bauchwand mit Peritonealknötchen und Drüsen in $\mathrm{M} u ̈ l l$ e - Formol eingelegt. - Wahrscheinl. tbc. Inf. d. d. Stb. (T).

Nr. 28: Staub in der Bauchhöhle; narbige Einziehungen in der etwas vergrösserten Milz; sonst 0 . B. - Nichts nachweisbar. N.

Nr. 29: Staub in sabkut. Abszess (hatte vor ca. 3 Wochen 2 gesunde Junge geboren); beiderseits grosse Milchdrüsen; vergrössert verhärtete Inguinaldrüsen ohne Erweichung; im Ausstrich des erbsengrossen Abszesses keine sicheren säurefesten Stäbcben; sonst o. B. im Antiforminzentrifugat des Abszessinhaltes viele schöne säurefeste Stäbchen; - tbc. Infektion d. d. Staub. T.

Milchdrüsen eingelegt.

Resultat: C.: S S E.: (T) N T.

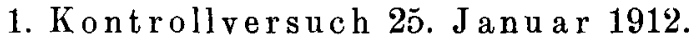

Kultur von kurzen Stäbchen und Diplokokken aus Peritonealexsudat 26, Bouillon $5 \mathrm{ccm}$ und $25 \%$ Antiformin $10 \mathrm{ccm}$; geschüttelt, nach $1 / 2$ Stunde zentrifugiert, gewaschen, zentrifugiert; Zentrifugat mit $15 \mathrm{ccm} \mathrm{0,9 \%}$ steriler $\mathrm{NaCl}$-Lösung 3 Meerschweinchen zu gleichen Teilen injiziert.

Nr. 30, weiss, m., 4', 8 schwarz.

Nr. 31, weiss, m., 10' gelb, 5, 6, 9 schwarz.

Nr. 32, gelb, w , 1, 2', 6', 7',11, 12 weiss, 8,13 schwarz. 
4. IV. Nr. 30-32 getötet. Sektionen:

Nr. 30 o. B. - N.

Nr. 31 Zystennieren; linsengrosser Lebertumor (beides eingelegt); sonst o. B. - N.

Nr. 32 Gravide, 3 ziemlich reife Föten; sonst o. B. - N. Resultat: N N N.

7. En. 1. Februar 1912.

Familienverbältnisse: $\mathrm{O}$. $\mathrm{B}$.

Wohnverhältnisse: 1 Schlafzimmer, 1 Wohnzimmer in einer Pension. Bewohner vollkommen gesund.

Raum: Eckzimmer, zweiter Stock nach Süden und Westen, hell und luftig. Ziemlich sauber, täg]ich geputzt.

540.330, Höhe 310. Fenster nach Süden $1: 2 \mathrm{~m}$, nach Westen 1,50:2 m.

Da nur sehr wenig Staub abgelagert ist und T B sehr nnwahrscheinlich, wird Untersuchung nach Cornet unterlassen.

Nach E.: Fussboden, Teppich, Polstermöbel.

Nach E.: 1. Febr. 1912.

Nr. 33 , gelb, w., $1,8,8^{\prime}, 9^{\prime}, 12,12^{\prime}, 13$ weiss, $2^{\prime}, 4^{\prime}, 4,5,6,5^{\prime}, 6^{\prime}, 7^{\prime}$ grau.

Nr. 34, weiss, w., 4 grau, $4^{\prime}, 5,5^{\prime}$ gelb.

Nr. 35, struppig schwarz, w., $1,9,9^{\prime}, 8^{\prime}, 12,12^{\prime}, 13$ weiss, $5^{\prime}, 8$ gelb.

6. III. Nr. $33-34$ getötet. Sektionen :

Nr. 33: Staub in der Bauchhöhle; sonst o. B. - N.

Nr. 34: Kein Staub nachweisbar; - es stellt sich später (am 4. IV.) heraus, dass eine Verwechselung mit 48 vorliegt; dabei ergibt dann 34 ausser Staub in der Bauchhöhle nichts besonderes. $-\mathrm{N}$.

Nr. 35: Gravide; zwei kleine Embryonen; Staub in der Bauchhöhle; sonst o. B. $-\mathrm{N}$.

Resultat: E. : N N N.

8. St. 12. Februar 1912.

Familienverhältnisse: Erwachsene und berufstätige Tochter aud Sohn.

Wohnverhältnisse: 2 kleine Räume mit 3 Betten und dunkle Küche, kalt nnd feucht, Mietpreis $18 \mathrm{Mk}$.

Krankengeschichte: 56 Jahre alt. Seit 2 Jahren in Behandlung. Rechts kalter Glutealabszess, auf zweimalige Punktion und Jodoformglyzerininjektion abgeheilt. Offene tuberkulöse Fistel am linken Knie. Wegen gleicher Affektionen wurden erfolgreich Gelenkresektionen an der linken Hand sowie an Hüfte und Knie ausgeführt.

Raum: Zu ebener Erde nach Osten, wegen Häuservorbau geg' $n$ Schlossberg steiler Lichteinfall, im Sommer nur eine Stande Sonne, mässig schmutzig.

275 hoch, 300 breit, 500 tief, 1 Fenster 124:92.

Schlaf-Wohnzimmer von meist bettlägeriger Pat. fast stets benutzt.

Nach Cornet: Türleiste, Bilder, Schrank, 2 qm Wand.

Nach E.: Fussboden, Bettrorleger.

Nach Cornet 12. Febr. 1912:

Nr. 36, struppig gelb, w., 4, 4', 11, 11' schwarz, 1, 13 weiss.

Nr. 37, weiss, w., 7-9 schwarz.

Nr. 38, schwarz, m., $7^{`}-9^{\star}, 12$ weiss. 
Nach E.: 13. Febr. 1912.

Nr. 39, weiss, m., $4^{\prime}, 6^{\prime}, 7^{\prime}, 10^{\prime}, 8,4$ schwarz und braun.

Nr. 40, schwarz, m., 6, 7, 9 weiss, 5, 13 gelb.

Nr. 41, schwarz, w., 1, 7-9, $7^{\prime}-9^{\prime}, 12^{\prime}$ weiss, 6, $6^{\prime}$ gelb.

14. II. Nr. 38 gestorben. Sektion: Staub in der Bauchhöhle; peritoneales Exsudat (viele Leukozyten und intrazelluläre Kokken); Leber und Milz matt, brüchig; sonst o. B. - Sept. Infektion. S.

19. II. Nr. 36 gestorben. Sektion: Hals angefressen, obere Thoraxapertur offen, Herz und Lungen feblen; Peritonealexsudat eitrig, Abdominalorgane schmierigfibrinös belegt; rechts im Becken haselnussgrosse Eiterung; Staub in der Bauchhöhle; multiple Nierenabszesse; Hänatome beiderseits am Nierenbilus; Milz wenig vergrössert; (im Ausstrich des Exsudates und des Abszesses Diplokokken); sonst o. B. - Sept. Infektion. S.

18. III. Nr. 37, 39-41 getötet. Sektionen :

Nr. 37: Staub in der Bauchhöhle; abgekapselter Leberabszess (im Ausstrich nur Leukozyten und wenige nicht säurefeste Stäbchen); Verwachsung zwischen Magen und Leber und mit der vorderen Bauchwand; sonst o. B. Überstandene sept. Infektion. ( $S$.)

Nr. 39 und 40: Staub in der Bauchhöhle; sonst o. B. N N.

Nr. 41: Gravide; 3 fast reife Föteu; Staub in der Bauchhöhle; sonst o. B. $-\mathrm{N}$.

Resultat: C.: S (S) S. E.: N N N.

9. Ka. 14. Februar 1912.

Familienverhältnisse: Erwachsene, berufstätige Tochter und deren ca. siebenjähriger Sohn, beide angeblich gesund.

Wohnverhältnisse: 1 Wohn-Schlafzimmer mit 1 Bett und 1 Schlafzimmer mit 2 Betten nach NNO, nur früh im Sommer etwas Sonne, und dunkle Küche auf den Hof. Mietpreis Mk. 27. -

Krankengeschichte: 63jähriger Kanstbildhauer. Seit Jan. 1911 an Bronchitis leidend. Im Febr. 1911 Pneumonie. Im Anschluss daran entwickelte sich rechte Oberlappenphthise mit viel Auswurf ohne Bazillenbefund.

Raum: Im dritten Stock nach NNO, enge Gasse, ziemlich steiler Lichteinfall, nur früh im Sommer etwas Sonne. Mässig schmutzig.

220 hoch, 445 breit, 400 tief; 2 Fenster à $106: 82$.

Schlaf-Wohnzimmer des Pat., von ihm fast stets benutzt.

Nach Cornet: Türleiste, mehrere Bilder, Uhr, 1 qm Wand am Bett.

Nach E.: Fussboden, Teppich.

Nach Cornet: 14. Febr. 1912.

Nr. 42, gran, m., 3 rot, $1-9$ weiss, $2-4$ gelb, $11-12$ graugelb.

Nr. 43 , weiss, w., 3 rot, $2,6,7,6^{\prime}, 7^{\prime}$ gelb, $2^{\prime}, 4^{\prime}$ grau.

Nr. 44, schwarz, m., 1, 6, 7, 6', 7’ gelb, 12 weiss.

Nach E.: 14. Febr. 1912.

Nr. 45, gelb, w., 1, 11, 12, 12 weiss, 2, 3, 5-7 schwarz.

Nr. 46, weiss, w., K. $8^{\prime}$ schwarz, 7, 8, 11' gelb:

Nr. 47, weiss, w., 3 rot, 2, 5-8 gelb, 4, 2', 4'-6' grau.

19. II. Nr. 43 gestorben. Sektion: Subkutanes Ödem; Milz hell verfärbt; Staub in der Bauchhöhle; Peritonealexsudat (im Ausstrich Diplokokken und kurze Stäbchen, wenig Leukozyten): sonst o. B. S. 
20. $11 \mathrm{Nr} .42$ gestorben. Sektion: wie 43 ; fibrinöse Peritonitis; Leberinfarkt; bronchopneumonische Herde in der rechten Lunge. S.

4. IV. Nr. 44-47 getötet: Sektionen :

Nr. 44: Staub in der Bauchhöhle; in der rechten oberen und linken mittleren Lunge infiltrierte Partien; Bronchialdrüsen vergrössert; sonst o. B. - Nichts nachweisbares. N.

Nr. 45: Subkutaner Abszess mit Staub (im Ausstrich einige säurefeste Stäbchen); Staub in der Bauchhöhle; barte vergrösserte Knoten inguinal, omental, mesenterial und mediastinal; Milz und Leber von zahlreichen Knötchen durchsetzt; Lungen: infiltrierte, verfärbte, deformierte Partien. - Tuberkulöse In fektion durch den Staub. T. Abszess, Milz, Teile von Leber, Lunge und Drüsen mit kochendem Wasser fixiert, in Alkohol eingelegt.

Nr. 46: Gravide, 2 mittelgrosse Föten; Staub in der Bauchböhle, sonst o. B. - Nichts nachweisbar. $N$.

Nr. 47: Staub in der Bauchhöhle; vergrösserte, narbig deformierte Milz mit abgekapseltem Abszess (im Ausstrich sehr feine, nicht srefste Diplobazillen); Netz, Peritoneum frei; einige nicht besonders harte, leicht vergrösserte Mesenterialknoten; Lungen etwa wie bei 45 ; hart vergrösserte Bronchialdrüsen. - Überstandene sept. Infektion und primäre Lungentuberkulose. (S.)

Lungenteile, Bronchialdrüsen wie bei 45 eingelegt.

Resultat: C.: S S N. E.: T N (S).

\section{Kontrollversuch am 1. März 1912.}

Aus Bouillonkultur von T B, typus humanus (Dr. B lasius), Öse ausgestrichen, gefärbt: schöne säurefeste Stäbchen. Nun fünf Ösen in $10 \mathrm{ccm}$ steriler $0,9 \% \mathrm{NaCl}$-Lösung verteilt und dieselbe zu gleichen Teilen injiziert.

Nr. 48, weiss, m., 1, 8, 12, 13 gelb, 2', $4^{\prime}-6^{\prime}$ grau.

Nr. 49, schwarz, w., $1-9$ weiss, 13 braun.

Bei der Sektion am 4. IV. wird 48 vermisst, an seiner Stelle 34 entdeckt, das ausser etwas Schmutz im Peritoneum nichts besonderes ergibt.

Nr. 49. Unter der Haut gut entwickelte Milchdrüsen (vor ca. 14 Tagen Geburt von 2 Jungen). Auf dem Peritoneum viele verstreute Knötchen. Netz knotig verdickt. Hart vergrösserte, teils käsig erweichte Mesenterialdrüsen. Milz und Leber vergrössert, höckerig von zahlreichen Knötchen durchsetzt. Abdomen sonst o. B. Lungen und Herz o. B.

Ausstrich aus Lymphdrüsenkäse: keine T B nachweisbar.

Eingelegt in Formol-Müller: R. Milchdrüse, Milz und mehrere Lymphknoten.

\section{Kontrollversuch 5. März 1912.}

$10 \mathrm{ccm}$ tbc. Sputum (im einfachen Ausstrich viele T B) mit $5 \mathrm{ccm} 50 \%$ Antiformin ca. $1 / 2$ Std. geschüttelt, zentrifugiert, mit 
steriler $0,9 \%$ NaCl-Lösung gewaschen, zentrifugiert, in $10 \mathrm{ccm}$ steriler $0,9 \% \mathrm{NaCl}-\mathrm{Lösung}$ intraperitoneal injiziert:

Nr. 50, struppig, schwarz, w., 2, 4, $2^{\prime} 4^{\prime}$ weiss, 11 schwarzgelb.

Nr. 51, gelb, w., 1, 4, 11' 12 weiss, $2^{\prime} 4^{\prime} 12^{\prime}$ schwarzgelb.

Am 12. März Nr. 51 gestorben. Sektion ergibt keinen besonderen Befund; jedoch Hals an- und Herz, Lungen herausgefressen.

Am 4. IV. Nr. 50 getötet. Sektion: Peritoneale Knötchen, knotige Verdickung von Netz und Gekröse mit käsig erweichtem Knoten (Ausstrich!); Milz vergrössert und unregelmässig von Knötchen durchsetzt. Sonst (auch Lunge) o. B.

Im Ausstrich der verkästen Drüse viele säurefeste Stäbchen.

\section{Resultatsuibersicht der Tierversuche.}

I. Vorversuch: antiforminbehandeltes tbc. Sputum 1 Tier inj. : T.

II. .Kontrollversuche:

1. antiforminbehandelte sept. Kultur

3 Tieren inj.: N N N.

2. T B-Kultur ohne weiteres

2 Tieren inj.: $\mathrm{O} \mathrm{T}$.

3. antiforminbehandeltes tbc. Sputum

2 Tieren inj.: OT.

III. Raumuntersuchungen:

C.:

\begin{tabular}{|c|c|c|}
\hline $\mathrm{Na}$. & $(S) \mathrm{N} \mathrm{S}$ & $(S)$ \\
\hline $\mathrm{Ke}$. & (S) $\mathrm{N} N$ & $\mathrm{~N}$ \\
\hline Ku. & $(S) \mathrm{S} S$ & $\mathrm{~N}$ \\
\hline Gu. & $\mathrm{S} \mathrm{S}(S) \mathrm{T}$ & $\mathrm{S}$ \\
\hline We. & $\mathrm{S} \mathrm{SO}$ & $(\mathrm{T})$ \\
\hline En. & --- & $\mathrm{N}$ \\
\hline St. & $\mathrm{S}(S) \mathrm{S}$ & $\mathrm{N}$ \\
\hline
\end{tabular}

Zeichenerklärung.

$\mathrm{T}$ bedeutet Tbc. mit Bazillenbefund

(T) , The. ohne Bazillenbefund

S " Tod an Sepsis

(S) \# überstandene Sepsis

N " nichts nachgewiesen

O $\quad$ missglückter Versuch

- $" \quad$ Versuch unterlassen.

Vergleichende Übersicht über III unter Fortlassung von En.:

Es fand sich

sept. Infektion

tbc. Infektion

keine Infektion bei Methode C.:

$16 \mathrm{mal}$

$1 \mathrm{mal}$

$4 \mathrm{mal}$ bei Methode E:

$4 \mathrm{mal}$

$8 \mathrm{mal}$

$9 \mathrm{mal}$. 
Die Methode nach E. zum Nachweis von T B im aspirablen Staub, welche theoretisch sowie nach den angestellten Laboratoriumsvorversuchen, aussichtsvoll erschien, hat sich also in den wenigen Fällen ihrer praktischen Anwendung bewährt.

Das rechtfertigt die Erwartung, dass auch sie bei weiterer Verbesserung dazu beitragen kann, die Ubiquitätsfrage sowie die Frage des Infektionsmodus der Tuberkulose, wie eingangs erwähnt, weiterer Klärung zuzuführen. Diesbezüglich aus den wenigen geschilderten Anwendungen Schlüsse zu ziehen, dürfte nicht berechtigt sein.

\section{Zusammenfassung.}

Auf die für das Problem der Inhalationstuberkulose wichtige Frage nach der Verbreitung der Tuberkelbazillen im aspirablen Staub konnten die bisherigen Staubuntersuchungen keine befriedigende Antwort geben, da sie 1. nicht den eigentlichen aspirablen Staub, der also im Moment der Untersuchung mit natürlichen Luftströmen in Mund oder Nase einzudringen vermag; sondern meist nur kleine, durch Abwischen und dgl. gewonnene Staubproben betrafen; da sie 2. nicht genügend empfindliche Nachweismethoden benutzten; denn a) war die Menge des Untersuchungsmaterials sehr klein und b) beeinträchtigten die neben den T B vorhandenen septischen Keime den Nachweis.

Um die Verbreitung der T B im aspirablen Staub festzustellen, musste also zunächst eine aussichtsreichere Nachweismethode gefunden werden. Den Inhalt der vorliegenden Arbeit bildet die A usarbeitung einer solchen Methode. Die mit Hilfe dieser Methode festzustellende tatsächliche Verbreitung der T B bleibt ausser Betracht bzw. späteren Untersuchungen vorbehalten.

Die neue Methode besteht in folgendem:

1. Reinigung des zu untersuchenden Raumes mit einem Staubsaugapparat, in dessen Saugrohr ein besonderes Staubauffangsystem eingeschaltet ist, welches den durch natürliche Luftströme aufwirbelbaren und aspirablen Staub in Wasser zurückhält.

2. Antiforminbehandlung des Staubwassers, dadurch Vernichtung der septischen Keime unter Schonung der T B, Auswaschen des Antiformins und Ausschaltung der rasch und ohne die vorhandenen $\mathrm{T} \mathrm{B}$ sedimentierenden Staubteile.

3. Injektion des restierenden Sedimentes in die Bauchhöhle gesunder Meerschweinchen.

Diese Methode, welche theoretisch und nach den angestellten Laboratoriumsvorversuchen aussichtsvoll erschien, wurde an einigen 
wenigen Fällen praktisch erprobt und zwar unter paralleler Anwendung der bisher besten Cornet schen Methode. Diese Anwendungsversuche, welche wegen ihrer geringen Zahl über die tatsächliche Verbreitung der TB nichts besagen wollen, sprechen für die Brauchbarkeit der Methode, so dass sich ihre weitere Nachprüfung verlohnen dürfte. Zur Untersuchung herangezogen wurden die Wohnräume von drei leidlich reinlichen offenen Phthisikern; das Resultat fiel nach der neuen Methode bei allen drei, nach der Cornetschen nur bei einem positiv aus. Bei einer reinlich behandelten offenen Gelerktuberkulose fanden sich nach beiden Methoden keine T B; ebensowenig in dem sauberen Wohnraum eines Gesunden. Bei einem unreinlichen offenen Phthisiker wurden mit der neuen Methode T B gefunden, während hier die Cornetschen Versuchstiere an Sepsis erkrankten; ebenso erging es mit den aus dem Kurssaal des pathologischen Instituts gewonnenen Staubproben. In dem zwar desinfizierten, aber von sehr unsauberen wenn auch gesunden Hinterbliebenen bewohnten Raum einer vor 4 Wochen verstorbenen offenen Phthise, wies die neue Methode T B nach, während von den Cornetschen Versuchstieren zwei gesund blieben und eines septisch erkrankte. - Im ganzen fanden sich:

Septische Infektionen bei der Cornetschen Methode 16 mal, bei der neuen Methode 4 mal.

Tuberkulöse Infektionen bei der Cornetschen Methode 1 mal, bei der neuen Methode 8 mal.

Keine nachweisbaren Infektionen bei der Cornetschen Methode 4 mal, bei der neuen Methode 9 mal.

Diese Resultate können natürlich nichts anderes beweisen, als dass der eingeschlagene Weg nicht aussichtslos ist. Da die neue Methode ziemlich umständlich und zeitraubend ist, wird es ihrer jahrelangen Anwendung bedürfen, um ein Material zu sammeln, welches zu weitergehenden Schlüssen berechtigt.

Zum Schlusse möchte ich nicht versäumen, allen denen, welche mich bei der Arbeit liebenswürdigst unterstützt haben, zu danken. Vor allem danke ich Herrn Professor Morawitz, auf dessen Anregung die ganze Arbeit zurückgeht, für seine vielfachen wertvollen Ratschläge und für die freundliche Überlassung des untersuchten Materials aus der Distriktspraxis der Freiburger medizinischen Poliklinik. Ferner habe ich für gütige Überlassung von Laboratoriumshilfsmitteln ausser Herrn Professor Morawitz, Herrn Geheimrat Schottelius, Herrn Geheimrat Aschoff und Herrn Professor Straub zu danken. 


\section{Literatur.}

1. Cornet, Die Tuberkulose. A. Holder, Wren, 1907, Bd. I, S. 1-6 und Literaturangaben ebenda S. 156-159.

2. Hanau und Schlenker, Virchows Arch. f. path. Anatom. u. Physiol, 134.

3. Naegeli, Virchows Arch. f. path. Anatom. u. Physiol., 140.

4. B u rkhardt, Zeitschr. f. Hygiene, 53.

5. H. Beitzke, Berlin. klin. Wochenschr, 1909, 9.

6. H a mb urger, Mitt. d. Ges. f. inn. Med. u. Kinderblk. Wien, 1909, 3.

7. Hamburger und Monti, Münchn. med. Wochenschr., 1909, 9.

8. C. Wegelin, Korr. f. Schweiz. Ärzte, 1910, 29.

9. Cornet, Zeitschr. f. Hygiene, 1888, 5 .

10. E. Krüger, Inaug.-Diss., Bonn, 1899.

11. A. Kastermann, Münchn. med. Wochenschr., 1891, S. 773.

12. Prausnitz, Münchn. med. Wochenschr., 1893, 1.

13. Petri, Arb. aus d. kais. Gesundheitsamt, 1893,

14. Kirchner, Zeitschr. f. Hygiene, 21.

15. Deipser, Korr. d. allg. ärztl. Ver. v. Thüringen, 1900, S. 513.

16. F. Gotschlich, Inaug.-Diss., Breslau, 1903.

17. F. W agner, Inaug.-Diss., Zürich, 1903.

18. B. He y m a n $n$, Zeitschr. f. Hygiene, 38.

19. Le Noire und Camus, Bull, de la soc. de biol, 18 Déc. 1908.

20. Dieselben, Presse médicale, 1909, 87.

21. Kö hli s c h, Zeitschr. f. Hygiene, 60 .

22. Cornet, Die Tuberkulose (siehe oben 1), Bd. I, S. 127.

23. Derselbe, ebenda Bd. I, S. 110.

24. Uhlenhuth, Beil. zu Bd. 42. Ref. d. Zentralbl. f. Bakt., S. 62.

25. K. Me yer, Tuberculosis VIII, 2, 1909.

26. Hü $\mathrm{n} \theta$, Hyg. Rundschau, 1908, 18.

27. Ra u, Hyg. Rundschau 69, 23.

28. Lagreye, Deutsche med. Wochenschr, 1910, 2.

29. Cornet, Die Tuberkulose, Bd. I, S. 108.

30. Kirstein, Zeitschr. f. Hygiene, 50, S. 186. 